TRANSACTIONS OF THE

AMERICAN MATHEMATICAL SOCIETY

Volume 365, Number 8, August 2013, Pages 4313-4350

S 0002-9947(2012)05778-3

Article electronically published on December 27, 2012

\title{
QUASI-INVARIANCE FOR HEAT KERNEL MEASURES \\ ON SUB-RIEMANNIAN INFINITE-DIMENSIONAL HEISENBERG GROUPS
}

\author{
FABRICE BAUDOIN, MARIA GORDINA, AND TAI MELCHER \\ To Len Gross
}

\begin{abstract}
We study heat kernel measures on sub-Riemannian infinitedimensional Heisenberg-like Lie groups. In particular, we show that CameronMartin type quasi-invariance results hold in this subelliptic setting and give $L^{p}$-estimates for the Radon-Nikodym derivatives. The main ingredient in our proof is a generalized curvature-dimension estimate which holds on approximating finite-dimensional projection groups. Such estimates were first introduced by Baudoin and Garofalo.
\end{abstract}

\section{Contents}

1. Introduction

1.1. Statement of results

1.2. Discussion of proofs

2. Functional inequalities on finite-dimensional Lie groups

2.1. Reverse inequalities under curvature bound assumptions

2.2. Wang type and integrated Harnack inequalities

3. Infinite-dimensional Heisenberg-like groups

3.1. Abstract Wiener spaces

3.2. Infinite-dimensional Heisenberg-like groups

3.3. Finite-dimensional projection groups

3.4. Derivatives and differential forms on $G$

3.5. Distances on $G_{C M}$

4. Infinite-dimensional computations

4.1. Curvature-dimension bounds and commutation relations

4.2. Functional inequalities on $G_{P}$

5. Heat kernel measure on $G$ and a quasi-invariance theorem

5.2. Quasi-invariance and Radon-Nikodym derivative estimates

Received by the editors August 12, 2011 and, in revised form, December 13, 2011. 2010 Mathematics Subject Classification. Primary 35K05, 43A15; Secondary 58J65. The first author's research was supported in part by NSF Grant DMS-0907326.

The second author's research was supported in part by NSF Grant DMS-1007496.

The third author's research was supported in part by NSF Grant DMS-0907293. 


\section{INTRODUCTION}

We prove Cameron-Martin type quasi-invariance results for subelliptic heat kernel measures on infinite-dimensional Heisenberg-like groups. These groups were first defined in [15] and quasi-invariance was proved for elliptic heat kernel measures in this setting. Quasi-invariance results are of interest, for example, in the study of smoothness of measures on infinite-dimensional spaces. In finite dimensions, one typically defines smoothness as absolute continuity with respect to some reference measure and smoothness of the associated density. In infinite dimensions, in the absence of a canonical reference measure, alternative interpretations of smoothness must be made; see for example [9, 14, 28, 29. In particular, in an infinite-dimensional setting, it is natural to interpret quasi-invariance as a smoothness property.

Quasi-invariance of heat kernel measures in infinite dimensions has previously been the subject of much study in elliptic settings; see for example the review [12. and the references therein. Typically the proofs in the elliptic case rely on lower bounds on the Ricci curvature (as was the case in [15]); of course, such lower bounds are unavailable in a subelliptic setting. To the authors' knowledge, this is the first quasi-invariance result in an infinite-dimensional subelliptic setting.

1.1. Statement of results. Let $(W, H, \mu)$ be an abstract Wiener space and let $\mathbf{C}$ be a finite-dimensional inner product space. Define $\mathfrak{g}=W \times \mathbf{C}$ to be an infinite-dimensional Heisenberg-like Lie algebra, which is constructed as an infinitedimensional step 2 nilpotent Lie algebra with continuous Lie bracket satisfying the following condition:

$$
[W, W]=\mathbf{C} .
$$

Let $G$ denote $W \times \mathbf{C}$, thought of as a group with operation

$$
g_{1} \cdot g_{2}=g_{1}+g_{2}+\frac{1}{2}\left[g_{1}, g_{2}\right]
$$

Then $G$ is a Lie group with Lie algebra $\mathfrak{g}$, and $G$ contains the subgroup $G_{C M}=$ $H \times \mathbf{C}$ which has Lie algebra $\mathfrak{g}_{C M}$. See Section 3.2 for definitions and details.

Now let $\left\{B_{t}\right\}_{t \geq 0}$ be a Brownian motion on $W$. The solution to the stochastic differential equation

$$
d g_{t}=g_{t} \circ d B_{t} \quad \text { with } g_{0}=e
$$

is a Brownian motion on $G$, which is defined explicitly in Proposition 5.1 and Definition 5.2. For all $t>0$, let $\nu_{t}=\operatorname{Law}\left(g_{2 t}\right)$ denote the heat kernel measure at time $2 t$. At this point, let us briefly comment that, as mentioned in [19], it may seem at first glance that the restriction $\operatorname{dim}(\mathbf{C})<\infty$ implies that this subelliptic example is in some sense only finitely many steps from being elliptic. However, this is truly a subelliptic model and the topologies one must deal with in this setting significantly change the standard analysis and introduce several non-trivial complications not present in the elliptic case. For further discussion, see Section 1.3 of [19].

The main results of the present paper are presented in Section 5.2. Namely, in Theorem [5.9] we prove that $\nu_{t}$ is quasi-invariant under translation by elements of $G_{C M}$ and obtain bounds on the $L^{p}$-norms of the Radon-Nikodym derivatives. Then given the equivalence of measures and the $L^{p}$-estimates on the associated densities, 
we are able to immediately prove in Corollary 5.11 that the semi-group is strong Feller on $G_{C M}$.

To put these results in context, again recall that if $W$ is finite-dimensional, then (1.1) implies that $\operatorname{span}\left\{\left(\xi_{i}, 0\right),\left[\left(\xi_{i}, 0\right),\left(\xi_{j}, 0\right)\right]\right\}=\mathfrak{g}$, where $\left\{\xi_{i}\right\}_{i=1}^{\operatorname{dim}(W)}$ is some orthonormal basis of $W$, and thus we have satisfaction of Hörmander's condition. This then implies that $\nu_{t}$ is a smooth measure, in the sense that $\nu_{t}$ is absolutely continuous with respect to Haar measure on $G=W \times \mathbf{C}$ and its density is a smooth function on $G$. If $W$ (and thus $G$ ) is infinite-dimensional, however, the notion of smoothness is not so well-defined, and quasi-invariance may be interpreted as a first step toward proving $\nu_{t}$ is a "strictly positive" smooth measure.

1.2. Discussion of proofs. Functional inequalities provide a powerful tool to study the problem of the equivalence of heat kernel measures. In particular, it is a well-known fact that, on a finite-dimensional complete Riemannian manifold $\mathbb{M}$ with non-negative Ricci curvature, the heat semi-group $\left\{P_{t}\right\}_{t \geq 0}$ satisfies the Harnack type inequality

$$
\left(P_{t} f\right)^{\alpha}(x) \leq P_{t} f^{\alpha}(y) \exp \left(\frac{\alpha}{\alpha-1} \frac{d^{2}(x, y)}{4 t}\right),
$$

where $x, y \in \mathbb{M}, f \in L^{\infty}(\mathbb{M})$ with $f \geq 0$, and $\alpha>1$ (see for example [34]). Using the above inequality with indicator functions immediately implies that the heat kernel measures $p_{t}(x, d z)$ and $p_{t}(y, d z)$ are equivalent. Of course, in a finitedimensional framework, the latter is obvious and may be seen from the positivity of the heat kernel. But the relevant fact here is that the functional inequality (1.2) is independent of the dimension of the manifold $\mathbb{M}$ and we may therefore hope that it holds even in some infinite-dimensional settings, where equivalence of measures is a highly non-trivial problem.

Again, a lower bound on the Ricci curvature of $\mathbb{M}$ typically plays a major role in the proof of inequalities like (1.2) and such bounds are unavailable in our subelliptic setting. However, in a recent work [8], Baudoin and Garofalo introduced a generalized curvature-dimension inequality that holds in a general class of subRiemannian settings. The main idea is to control sub-Riemannian curvature quantities both in the horizontal and the vertical directions. In the present paper, we prove that a uniform generalized curvature-dimension inequality holds on appropriate finite-dimensional approximation groups of $G$, and that, as a consequence, a uniform version of (1.2) holds on the finite-dimensional approximation groups. A by-product of this is a Cameron-Martin type quasi-invariance result for the heat kernel measure on $G$.

Let us emphasize that our approach is actually quite general and does not rely on the specific nature of the infinite-dimensional Heisenberg-like groups we consider here. The main ingredient is the existence of good finite-dimensional approximations on which uniform generalized curvature-dimension bounds hold. As was done in [16] in the elliptic setting, one could significantly generalize the method to include other infinite-dimensional subelliptic settings.

The organization of the paper is briefly as follows. In Section 2 we review results for subelliptic heat kernels on finite-dimensional Lie groups under the assumption of generalized curvature-dimension estimates and satisfaction of certain commutation relations. In particular, in Section 2.1, we show that under these assumptions reverse Poincaré and log Sobolev estimates hold, and in Section 2.2 we show how 
these estimates in turn give Wang type and integrated Harnack inequalities. The ideas in this section in a finite-dimensional setting are discussed in more detail and in greater generality in [6].

In Section 3 we review the definition of the infinite-dimensional Heisenberglike groups first considered in [15]. In the present paper, we choose a topological structure that is better adapted to subellipticity, and in this way our construction parallels [19], where subelliptic heat kernel measure was also studied. In this section, we also review the properties of infinite-dimensional Heisenberg-like groups required for the sequel, as well as recalling the Cameron-Martin subgroup and the finitedimensional projection groups which serve as approximations.

In Section 4.1 we show that the curvature-dimension estimates and commutation relations considered in Section 2 are satisfied on the infinite-dimensional Heisenberglike groups and their finite-dimensional projections, and thus in Section 4.2 we are able to show that reverse Poincaré and log Sobolev inequalities, as well as Wang type Harnack inequalities, hold on the finite-dimensional projection groups.

Finally, in Section 5.1 we review the construction of subelliptic heat kernel measures on the infinite-dimensional Heisenberg-like groups, and in Section 5.2 we show how the Wang type Harnack inequalities proved in Section 4.2 for the finitedimensional projection groups imply quasi-invariance of the subelliptic heat kernel measure under translation by elements of the Cameron-Martin space, as well as yielding $L^{p}$-estimates for the Radon-Nikodym derivatives. We also show how the quasi-invariance and $L^{p}$-estimates then immediately imply that $P_{t}$ is strong Feller on $G_{C M}$.

\section{Functional inequalities on finite-Dimensional Lie Groups}

For this entire section, $G$ will denote a real finite-dimensional connected unimodular Lie group with Lie algebra $\mathfrak{g}$ and identity element $e$. Let $d x$ denote the bi-invariant Haar measure on $G$. For $x \in G$, let $L_{x}$ and $R_{x}$ denote the left and right translation by $x$, respectively. For $A \in \mathfrak{g}$, let $\tilde{A}$ denote the unique left invariant vector field on $G$ such that $\tilde{A}(e)=A \in \mathfrak{g}$, that is, $\tilde{A}(g)=L_{x *} A$.

We will fix a linearly independent collection $\left\{X_{i}\right\}_{i=1}^{n} \subset \mathfrak{g}$ for which there exists some $r$ such that

$$
\begin{aligned}
\mathfrak{g}=\operatorname{span}\left\{X_{i_{1}},\left[X_{i_{1}}, X_{i_{2}}\right], \ldots,\left[X_{i_{1}},\left[X_{i_{2}}, \ldots,\left[X_{i_{r-1}}, X_{i_{r}}\right] \ldots\right]\right]:\right. \\
\left.i_{1}, \ldots, i_{r}=1, \ldots, n\right\} .
\end{aligned}
$$

We will refer to $\mathcal{H}:=\operatorname{span}\left(\left\{X_{i}\right\}_{i=1}^{n}\right)$ as the horizontal directions, and we will suppose that $\mathfrak{g}$ is equipped with an inner product for which $\left\{X_{i}\right\}_{i=1}^{n}$ is an orthonormal basis of $\mathcal{H}$. In particular, (2.1) implies that $\left\{\tilde{X}_{i}\right\}_{i=1}^{n}$ is a Hörmander set of vector fields in the sense that the $\tilde{X}_{i}$ 's and all their commutators up to order $r$ generate $T_{g} G$ for all $g \in G$. Thus, by the classical Hörmander's theorem [23] the second order differential operator

$$
L=\sum_{i=1}^{n} \tilde{X}_{i}^{2}
$$

is hypoelliptic, and, for all $t>0$, there exists a smooth kernel $p_{t}: G \times G \rightarrow \mathbb{R}^{+}$so that

$$
P_{t} f(x):=e^{t \bar{L}} f(x)=\int_{G} f(y) p_{t}(x, y) d y,
$$


for all $f \in L^{2}(G, d y)$, where $\bar{L}$ denotes the $L^{2}(G, d y)$ closure of $\left.L\right|_{C_{c}^{\infty}(G)}$. The heat semi-group $\left\{P_{t}\right\}_{t>0}$ is a symmetric Markov semi-group, and, since $p_{t}$ is smooth, the mapping $(t, x) \mapsto P_{t} f(x)$ is smooth on $(0, \infty) \times G$ for any $f \in L^{p}(d x), p \in[1, \infty]$.

In a slight abuse of notation, we will write that $p_{t}(e, x)=p_{t}(x)$. In particular, the left invariance of $L$ implies that $p_{t}$ is a left convolution kernel and thus $p_{t}(x, y)=$ $p_{t}\left(x y^{-1}\right)$. We call the measure $p_{t} d y$ the heat kernel measure on $G$ associated to $L$. By construction, the operator $P_{t}$ commutes with left translations, and since our Lie group is unimodular the Haar measure is bi-invariant. Thus, $p_{t}(x, y)=p_{t}\left(e, x^{-1} y\right)$. Since it is additionally known that $p_{t}$ is a symmetric kernel, we have the following lemma of some standard properties of the heat kernel, which we state without proof.

Lemma 2.1. For all $t>0$ and $x, y \in G$,

(1) $p_{t}(x, y)=p_{t}\left(x^{-1} y\right)=p_{t}\left(y^{-1} x\right)$ and

(2) $p_{t}\left(x^{-1}\right)=p_{t}(x)$.

Another well-known interpretation of the heat kernel measure $p_{t} d y$ is the distribution at time $2 t$ of Brownian motion on $G$. For $\left\{b_{i}\right\}_{i=1}^{n}$ independent real-valued Brownian motions, $b_{t}=\sum_{i=1}^{n} b_{t}^{i} X_{i}$ is a Brownian motion on $\mathcal{H}$. Now for $t>0$ let $g_{t}$ denote the solution to the following Stratonovitch stochastic differential equation:

$$
d g_{t}=g_{t} \circ d b_{t}:=L_{g_{t} *} \circ d b_{t}=\sum_{i=1}^{n} \tilde{X}\left(g_{t}\right) \circ d b_{t}^{i}, \quad \text { with } g_{0}=e .
$$

Then, $\left\{g_{t}\right\}_{t \geq 0}$ is a Brownian motion on $G$ started at the identity, and, for all $t>0$, $\operatorname{Law}\left(g_{t}\right)=p_{t / 2} d y$.

For $f, g \in C^{\infty}(G)$, we define the standard differential forms

$$
\begin{aligned}
\Gamma(f, g) & :=\frac{1}{2}(L(f g)-f L g-g L f)=\sum_{j=1}^{n}\left(\tilde{X}_{i} f\right)\left(\tilde{X}_{i} g\right) \text { and } \\
\Gamma_{2}(f, g) & :=\frac{1}{2}(L \Gamma(f, g)-\Gamma(f, L g)-\Gamma(g, L f)) .
\end{aligned}
$$

We follow the usual notational convention that $\Gamma(f):=\Gamma(f, f)$ and $\Gamma_{2}(f):=$ $\Gamma_{2}(f, f)$. We will also need to consider derivatives in non-horizontal, or vertical, directions. Let $\mathcal{V} \subset \mathfrak{g}$ denote the Lie subalgebra such that we have the orthogonal decomposition (with respect to the inner product on $\mathfrak{g}$ )

$$
\mathfrak{g}=\mathcal{H} \oplus \mathcal{V},
$$

and let $\left\{Z_{\ell}\right\}_{\ell=1}^{N}$ denote an orthonormal basis of $\mathcal{V}$, where $N=\operatorname{dim}(\mathcal{V})$. Following [2] and [8] we define

$$
\begin{aligned}
\Gamma^{Z}(f, g) & :=\sum_{\ell=1}^{N}\left(\tilde{Z}_{\ell} f\right)\left(\tilde{Z}_{\ell} g\right) \text { and } \\
\Gamma_{2}^{Z}(f, g) & :=\frac{1}{2}\left(L \Gamma^{Z}(f, g)-\Gamma^{Z}(f, L g)-\Gamma^{Z}(g, L f)\right) .
\end{aligned}
$$

Again we will let $\Gamma^{Z}(f):=\Gamma^{Z}(f, f)$ and $\Gamma_{2}^{Z}(f):=\Gamma_{2}^{Z}(f, f)$.

2.1. Reverse inequalities under curvature bound assumptions. In this section we consider some implications of certain curvature bound assumptions on $G$. These curvature bounds first appeared in [8] and serve as generalizations of the standard curvature-dimension inequalities which appear in the elliptic literature 
(see for example [3, 4, [5, 26] and the references therein). The assumption may be stated as follows: Suppose that there exist $\alpha, \beta>0$ such that, for any $\nu>0$ and $f \in C^{\infty}(G)$,

$$
\Gamma_{2}(f)+\nu \Gamma_{2}^{Z}(f) \geq \alpha \Gamma^{Z}(f)-\frac{\beta}{\nu} \Gamma(f) .
$$

Before proceeding with the primary results of this section, we prove the following lemma which will be helpful in the sequel.

Lemma 2.2. Suppose $\phi: J \rightarrow \mathbb{R}$ is a smooth function on an open interval $J \subset \mathbb{R}$ and $f: G \rightarrow \mathbb{R}$ is a measurable function. Fix $T>0$ and set

$$
\Sigma(t, x)=P_{t}\left(\phi\left(P_{T-t} f\right)\right)(x),
$$

for all $t \in[0, T]$ and $x \in G$ (assuming $\left.P_{T-t} f(G) \subset J\right)$. Then

$$
\frac{d \Sigma}{d t}=P_{t}\left(\phi^{\prime \prime}\left(P_{T-t} f\right) \Gamma\left(P_{T-t} f\right)\right)(x) .
$$

Proof. For simplicity, set $u_{t}=P_{T-t} f$. Then we just compute

$$
\frac{d \Sigma}{d t}=P_{t}\left(L \phi\left(u_{t}\right)+\frac{d}{d t} \phi\left(u_{t}\right)\right)=P_{t}\left(L \phi\left(u_{t}\right)-\phi^{\prime}\left(u_{t}\right) L u_{t}\right) .
$$

Note that

$$
\begin{aligned}
L\left(\phi\left(u_{t}\right)\right) & =\sum_{i=1}^{n} \tilde{X}_{i}^{2}\left(\phi\left(u_{t}\right)\right)=\sum_{i=1}^{n} \tilde{X}_{i}\left(\phi^{\prime}\left(u_{t}\right)\left(\tilde{X}_{i} u_{t}\right)\right) \\
& =\sum_{i=1}^{n}\left(\phi^{\prime \prime}\left(u_{t}\right)\left(\tilde{X}_{i} u_{t}\right)^{2}+\phi^{\prime}\left(u_{t}\right)\left(\tilde{X}_{i}^{2} u_{t}\right)\right)=\phi^{\prime \prime}\left(u_{t}\right) \Gamma\left(u_{t}\right)+\phi^{\prime}\left(u_{t}\right) L u_{t} .
\end{aligned}
$$

Then combining this with (2.3) yields the desired result.

We now prove that, assuming the curvature bound stated above, a reverse Poincaré inequality holds on $G$.

Notation 2.3. Let $\mathcal{C}$ denote the set of functions $f: G \rightarrow \mathbb{R}$ such that $f \in C^{\infty}(G) \cap$ $L^{\infty}(G)$ and $f, \sqrt{\Gamma(f)}, \sqrt{\Gamma^{Z}(f)} \in L^{2}(G)$. Note for example that $\mathcal{C}$ includes all smooth functions with compact support. We will also let $\mathcal{C}^{+}$denote functions $f$ such that $f=g+\varepsilon$ for some $g \in \mathcal{C}$ with $g \geq 0$ and $\varepsilon>0$.

Remark 2.4. It is shown in 8 ] that these function spaces are stable under $P_{t}$. That is, if $f \in \mathcal{C}$, then $P_{t} f \in \mathcal{C}$ for all $t>0$, and similarly for $\mathcal{C}^{+}$.

Proposition 2.5. Assume that a curvature bound is satisfied as stated above in (2.2). Then, for all $T>0$ and $f \in \mathcal{C}$,

$$
\Gamma\left(P_{T} f\right)+\alpha T \Gamma^{Z}\left(P_{T} f\right) \leq \frac{1+\frac{2 \beta}{\alpha}}{2 T}\left(P_{T}\left(f^{2}\right)-\left(P_{T} f\right)^{2}\right) .
$$

In particular, this implies that

$$
\Gamma\left(P_{T} f\right) \leq \frac{1+\frac{2 \beta}{\alpha}}{2 T}\left(P_{T}\left(f^{2}\right)-\left(P_{T} f\right)^{2}\right) .
$$


Proof. For $t \in[0, T]$, define the functional

$$
\Phi(t)=a(t) P_{t}\left(\Gamma\left(P_{T-t} f\right)\right)+b(t) P_{t}\left(\Gamma^{Z}\left(P_{T-t} f\right)\right),
$$

where $a, b:[0, T] \rightarrow[0, \infty)$ are control functions yet to be chosen. A straightforward computation shows that

$$
\begin{aligned}
\Phi^{\prime}(t)=a^{\prime}(t) P_{t}\left(\Gamma\left(P_{T-t} f\right)\right)+b^{\prime}(t) P_{t}\left(\Gamma^{Z}\left(P_{T-t} f\right)\right) \\
+2 a(t) P_{t}\left(\Gamma_{2}\left(P_{T-t} f\right)\right)+2 b(t) P_{t}\left(\Gamma_{2}^{Z}\left(P_{T-t} f\right)\right),
\end{aligned}
$$

and the inequality (2.2) implies that

$$
\Gamma_{2}\left(P_{T-t} f\right)+\frac{b(t)}{a(t)} \Gamma_{2}^{Z}\left(P_{T-t} f\right) \geq-\frac{\beta a(t)}{b(t)} \Gamma\left(P_{T-t} f\right)+\alpha \Gamma^{Z}\left(P_{T-t} f\right) .
$$

Thus,

$$
\Phi^{\prime} \geq\left(a^{\prime}-2 \beta \frac{a^{2}}{b}\right) P_{t}\left(\Gamma\left(P_{T-t} f\right)\right)+\left(b^{\prime}+2 \alpha a\right) P_{t}\left(\Gamma^{Z}\left(P_{T-t} f\right)\right) .
$$

We now choose the functions $a$ and $b$ so that

$$
b^{\prime}+2 \alpha a=0
$$

and

$$
a^{\prime}-2 \beta \frac{a^{2}}{b}=C,
$$

where $C$ is a constant independent of $t$. This leads to the candidates

$$
a(t)=\frac{1}{\alpha}(T-t)
$$

and

$$
b(t)=(T-t)^{2} .
$$

For this choice of $a$ and $b$, the inequality (2.4) becomes

$$
\Phi^{\prime}(t) \geq-\frac{1}{\alpha}\left(1+\frac{2 \beta}{\alpha}\right) P_{t}\left(\Gamma\left(P_{T-t} f\right)\right) .
$$

By Lemma 2.2 with $\phi(x)=x^{2}$, we have that

$$
\frac{d}{d t} P_{t}\left(P_{T-t} f\right)^{2}=2 P_{t}\left(\Gamma\left(P_{T-t} f\right)\right)
$$

and thus integrating (2.5) from 0 to $T$ yields the desired result.

Under an additional assumption, the curvature bound (2.2) also implies a reverse log Sobolev type inequality which is much stronger than the previous reverse Poincaré inequality. The additional required assumption here is the following commutation: for any $f \in C^{\infty}(G)$,

$$
\Gamma\left(f, \Gamma^{Z}(f)\right)=\Gamma^{Z}(f, \Gamma(f)) .
$$

First we prove the following lemma given this assumption.

Lemma 2.6. For fixed $T>0, x \in G$, and $f \in \mathcal{C}$, define the entropy functionals

$$
\Phi_{1}(t)=P_{t}\left(\left(P_{T-t} f\right) \Gamma\left(\ln P_{T-t} f\right)\right)(x)
$$

and

$$
\Phi_{2}(t)=P_{t}\left(\left(P_{T-t} f\right) \Gamma^{Z}\left(\ln P_{T-t} f\right)\right)(x),
$$


for $t \in[0, T]$. Then, assuming (2.6) holds,

$$
\Phi_{1}^{\prime}(t)=2 P_{t}\left(\left(P_{T-t} f\right) \Gamma_{2}\left(\ln P_{T-t} f\right)\right)(x)
$$

and

$$
\Phi_{2}^{\prime}(t)=2 P_{t}\left(\left(P_{T-t} f\right) \Gamma_{2}^{Z}\left(\ln P_{T-t} f\right)\right)(x) .
$$

Proof. For $t \in[0, T]$ and $x \in G$, consider the functionals

$$
\phi_{1}(t, x)=\left(P_{T-t} f\right)(x) \Gamma\left(\ln P_{T-t} f\right)(x)
$$

and

$$
\phi_{2}(t, x)=\left(P_{T-t} f\right)(x) \Gamma^{Z}\left(\ln P_{T-t} f\right)(x) .
$$

If we prove that

$$
L \phi_{1}+\frac{\partial \phi_{1}}{\partial t}=2\left(P_{T-t} f\right) \Gamma_{2}\left(\ln P_{T-t} f\right)
$$

and

$$
L \phi_{2}+\frac{\partial \phi_{2}}{\partial t}=2\left(P_{T-t} f\right) \Gamma_{2}^{Z}\left(\ln P_{T-t} f\right),
$$

then the result follows almost immediately.

Again for simplicity, take $u(t, x)=P_{T-t} f(x)$ and here let $u_{t}=\frac{\partial u}{\partial t}$. Then a simple computation gives

$$
\frac{\partial \phi_{1}}{\partial t}=u_{t} \Gamma(\ln u)+2 u \Gamma\left(\ln u, \frac{u_{t}}{u}\right) .
$$

On the other hand,

$$
L \phi_{1}=L u \Gamma(\ln u)+u L \Gamma(\ln u)+2 \Gamma(u, \Gamma(\ln u)) .
$$

Combining these equations we obtain

$$
L \phi_{1}+\frac{\partial \phi_{1}}{\partial t}=u L \Gamma(\ln u)+2 \Gamma(u, \Gamma(\ln u))+2 u \Gamma\left(\ln u, \frac{u_{t}}{u}\right) .
$$

We now see that

$$
\begin{aligned}
2 u \Gamma_{2}(\ln u) & =u(L \Gamma(\ln u)-2 \Gamma(\ln u, L(\ln u))) \\
& =u L \Gamma(\ln u)-2 u \Gamma(\ln u, L(\ln u)) .
\end{aligned}
$$

Observing that

we may conclude

$$
L(\ln u)=-\frac{\Gamma(u)}{u^{2}}-\frac{u_{t}}{u},
$$

$$
L \phi_{1}+\frac{\partial \phi_{1}}{\partial t}=2\left(P_{T-t} f\right) \Gamma_{2}\left(\ln P_{T-t} f\right) .
$$

In the same vein, we obtain

$$
L \phi_{2}+\frac{\partial \phi_{2}}{\partial t}=u L \Gamma^{Z}(\ln u)+2 \Gamma\left(u, \Gamma^{Z}(\ln u)\right)+2 u \Gamma^{Z}\left(\ln u, \frac{u_{t}}{u}\right) .
$$

This time, using the definition of $\Gamma_{2}^{Z}$, we find

$$
\begin{aligned}
2 u \Gamma_{2}^{Z}(\ln u) & =u\left(L \Gamma^{Z}(\ln u)-2 \Gamma^{Z}(\ln u, L(\ln u))\right) \\
& =u L \Gamma^{Z}(\ln u)+2 u \Gamma^{Z}\left(\ln u, \frac{\Gamma(u)}{u^{2}}\right)+2 u \Gamma^{Z}\left(\ln u, \frac{u_{t}}{u}\right) .
\end{aligned}
$$


From this last equation it is now clear that under assumption (2.6) we have

$$
L \phi_{2}+\frac{\partial \phi_{2}}{\partial t}=2 u \Gamma_{2}^{Z}(\ln u)
$$

and this concludes the proof.

Given this lemma, we may now prove the following reverse log Sobolev inequality holds.

Theorem 2.7. Suppose that (2.2) and (2.6) are satisfied. Then, for any $T>0$ and $f \in \mathcal{C}^{+}$,

$$
\Gamma\left(\ln P_{T} f\right)+\alpha T \Gamma^{Z}\left(\ln P_{T} f\right) \leq \frac{1+\frac{2 \beta}{\alpha}}{T}\left(\frac{P_{T}(f \ln f)}{P_{T} f}-\ln P_{T} f\right) .
$$

In particular, the following reverse log Sobolev inequality holds:

$$
\Gamma\left(\ln P_{T} f\right) \leq \frac{1+\frac{2 \beta}{\alpha}}{T}\left(\frac{P_{T}(f \ln f)}{P_{T} f}-\ln P_{T} f\right) .
$$

Proof. For $t \in[0, T]$, we define the functional

$$
\Psi(t)=a(t) \Phi_{1}(t)+b(t) \Phi_{2}(t),
$$

where $\Phi_{1}$ and $\Phi_{2}$ are as defined in Lemma 2.6 and $a, b$ are non-negative control functions to be chosen later. Since we assume (2.6) holds, Lemma 2.6 implies that

$$
\begin{aligned}
\Psi^{\prime}(t)= & a^{\prime}(t) \Phi_{1}(t)+b^{\prime}(t) \Phi_{2}(t) \\
& +2 a(t) P_{t}\left(\left(P_{T-t} f\right) \Gamma_{2}\left(\ln P_{T-t} f\right)\right)+2 b(t) P_{t}\left(\left(P_{T-t} f\right) \Gamma_{2}^{Z}\left(\ln P_{T-t} f\right)\right) .
\end{aligned}
$$

Thus, given the curvature bound and working exactly as in Proposition 2.5, we are led to the same choices,

$$
a(t)=\frac{1}{\alpha}(T-t) \quad \text { and } \quad b(t)=(T-t)^{2} .
$$

For this choice of $a$ and $b$, we have the inequality

$$
\Psi^{\prime}(t) \geq-\frac{1}{\alpha}\left(1+\frac{2 \beta}{\alpha}\right) \Phi_{1}(t) .
$$

Taking $\phi(x)=x \ln x$ in Lemma 2.2 implies that

$$
\begin{aligned}
\frac{d}{d t} P_{t}\left(\left(P_{T-t} f\right)\left(\ln P_{T-t} f\right)\right) & =P_{t}\left(\frac{\Gamma\left(P_{T-t} f\right)}{P_{T-t} f}\right) \\
& =P_{t}\left(\left(P_{T-t} f\right) \Gamma\left(\ln P_{T-t} f\right)\right)=\Phi_{1}(t),
\end{aligned}
$$

and thus integrating (2.7) from 0 to $T$ then yields the claimed result.

Remark 2.8. Other choices of control functions could be made to satisfy the desired criteria in the proofs of Proposition 2.5] and Theorem 2.7. In particular, we could have taken

$$
a(t)=\frac{1}{\alpha}((1+\delta) T-t)
$$

and

$$
b(t)=((1+\delta) T-t)^{2},
$$


for any $\delta \geq 0$. This choice of $a$ and $b$ would give the following generalized estimates. The following would generalize Proposition 2.5. for all $T>0$ and $f \in L^{\infty}(G)$,

$$
\begin{aligned}
\Gamma\left(P_{T} f\right) & +\alpha(1+\delta) T \Gamma^{Z}\left(P_{T} f\right) \\
\leq & \frac{1+\frac{2 \beta}{\alpha}}{2(1+\delta) T}\left(P_{T}\left(f^{2}\right)-\left(P_{T} f\right)^{2}\right)+\frac{\delta}{1+\delta} P_{T}(\Gamma(f))+\frac{\alpha \delta^{2}}{1+\delta} T P_{T}\left(\Gamma^{Z}(f)\right) .
\end{aligned}
$$

Also, the following statement would generalize Theorem 2.7 for all $T>0$ and $f \in \mathcal{C}$,

$$
\begin{aligned}
P_{T} f \Gamma\left(\ln P_{T} f\right)+\alpha(1+\delta) T P_{T} f \Gamma^{Z}\left(\ln P_{T} f\right) \\
\leq \frac{1+\frac{2 \beta}{\alpha}}{(1+\delta) T}\left(P_{T}(f \ln f)-\left(P_{T} f\right) \ln P_{T} f\right) \\
\quad+\frac{\delta}{1+\delta} P_{T}(f \Gamma(\ln f))+\frac{\alpha \delta^{2}}{1+\delta} T P_{T}\left(f \Gamma^{Z}(\ln f)\right) .
\end{aligned}
$$

Such generalized estimates have been used in [7] to prove lower bounds for the heat kernel.

2.2. Wang type and integrated Harnack inequalities. A reverse log Sobolev inequality such as in Theorem 2.7 is sufficient to prove an analogue of Wang's dimension-free Harnack inequality. Estimates of this type were first proved by Wang in a Riemannian setting under the assumption of a lower bound on the Ricci curvature [34]. Before stating the estimate, we must make the following definition.

Notation 2.9 (Horizontal distance). $\quad$ (1) The length of a $C^{1}$-path $\sigma:[a, b] \rightarrow G$ is defined as

$$
\ell(\sigma):=\int_{a}^{b}\left|L_{\sigma^{-1}(s) *} \dot{\sigma}(s)\right|_{\mathfrak{g}} d s .
$$

(2) A $C^{1}$-path $\sigma:[a, b] \rightarrow G$ is horizontal if $L_{\sigma(t)^{-1}{ }_{*}} \dot{\sigma}(t) \in \mathcal{H} \times\{0\}$ for a.e. $t$. Let $C^{1, h}$ denote the set of horizontal paths $\sigma:[0,1] \rightarrow G$.

(3) The horizontal distance between $x, y \in G$ is defined by

$$
d(x, y):=\inf \left\{\ell(\sigma): \sigma \in C^{1, h} \text { such that } \sigma(0)=x \text { and } \sigma(1)=y\right\} .
$$

For references on horizontal distance, or more general sub-Riemannian geometry, see for example [20, 31] and the references contained therein.

Proposition 2.10. Suppose there exists a constant $C<\infty$ such that, for all $T>0$ and $f \in \mathcal{C}^{+}$,

$$
\Gamma\left(\ln P_{T} f\right) \leq \frac{C}{T}\left(\frac{P_{T}(f \ln f)}{P_{T} f}-\ln P_{T} f\right) .
$$

Then, for all $T>0, x, y \in G, f \in L^{\infty}(G)$ with $f \geq 0$, and $p \in(1, \infty)$,

$$
\left(P_{T} f\right)^{p}(x) \leq P_{T} f^{p}(y) \exp \left(\frac{C p}{p-1} \frac{d^{2}(x, y)}{4 T}\right) .
$$

Proof. First take $f \in \mathcal{C}^{+}$. Let $b(s)=1+(p-1) s$ for $s \in[0,1]$ and $\sigma:[0,1] \rightarrow G$ be an arbitrary horizontal $C^{1}$-path such that $\sigma(0)=x$ and $\sigma(1)=y$. Define the functional

$$
\phi(s)=\frac{p}{b(s)} \ln P_{T} f^{b(s)}(\sigma(s)), \quad \text { for } s \in[0,1] .
$$


Differentiating $\phi$ with respect to $s$ and applying (2.8) yields

$$
\begin{aligned}
\phi^{\prime}(s)=- & \frac{p b^{\prime}(s)}{b(s)^{2}} \ln P_{T} f^{b(s)}(\sigma(s))+\frac{p}{b(s)}\left(\frac{d}{d s} \ln P_{T} f^{b(s)}\right)(\sigma(s)) \\
& \quad+\frac{p}{b(s)}\left\langle\left(d\left(\ln P_{T} f^{b(s)}\right)\right)(\sigma(s)), \sigma^{\prime}(s)\right\rangle \\
= & \frac{p(p-1)}{b(s)^{2}}\left(\frac{P_{T}\left(f^{b(s)} \ln f^{b(s)}\right)(\sigma(s))}{P_{T} f^{b(s)}(\sigma(s))}-\ln P_{T} f^{b(s)}(\sigma(s))\right) \\
& \quad+\frac{p}{b(s)}\left\langle d\left(\ln P_{T} f^{b(s)}\right)(\sigma(s)), \sigma^{\prime}(s)\right\rangle \\
\geq & \frac{p(p-1) T}{b(s)^{2} C} \Gamma\left(\ln P_{T} f^{b(s)}\right)(\sigma(s))+\frac{p}{b(s)}\left\langle d\left(\ln P_{T} f^{b(s)}\right)(\sigma(s)), \sigma^{\prime}(s)\right\rangle .
\end{aligned}
$$

Now, for every $\lambda>0$,

$$
\left\langle d\left(\ln P_{T} f^{b(s)}\right)(\sigma(s)), \sigma^{\prime}(s)\right\rangle \geq-\frac{1}{2 \lambda} \Gamma\left(\ln P_{T} f^{b(s)}\right)(\sigma(s))-\frac{\lambda}{2}\left|\sigma^{\prime}(s)\right|^{2},
$$

since $\sigma$ being horizontal implies that $\sigma^{\prime}(s) \in \operatorname{span}\left\{\tilde{X}_{i}(\sigma(s))\right\}_{i=1}^{n}$. In particular, choosing

$$
\lambda=\frac{C}{2(p-1) T} b(s)
$$

gives

$$
\phi^{\prime}(s) \geq-\frac{C p}{4(p-1) T}\left|\sigma^{\prime}(s)\right|^{2} .
$$

Integrating this inequality from 0 to 1 yields

$$
\ln P_{T} f^{p}(y)-\ln \left(P_{T} f\right)^{p}(x) \geq-\frac{C p}{4(p-1) T} \int_{0}^{1}\left|\sigma^{\prime}(s)\right|^{2} d s .
$$

Minimizing $\int_{0}^{1}\left|\sigma^{\prime}(s)\right|^{2} d s$ over the set of horizontal paths such that $\sigma(0)=x$ and $\sigma(1)=y$ shows that (2.9) holds for $f \in \mathcal{C} \cap C^{\infty}(G)$.

To prove the estimate for general $f \geq 0$, let $C^{\infty}(G) \ni h_{n} \geq 0$ be an increasing sequence of functions with compact support such that $h_{n} \uparrow 1$. Then (2.9) holds for $g=h_{n} P_{\tau} f+\varepsilon \in \mathcal{C} \cap C^{\infty}(G)$ for all $n, \tau>0$, and $\varepsilon>0$. Then letting $\varepsilon \rightarrow 0, \tau \rightarrow 0$, and $n \rightarrow \infty$ in the inequality completes the proof of (2.9) for $f \geq 0$.

Wang type Harnack inequalities in turn are equivalent to so-called "integrated Harnack inequalities" as in the following lemma. Here we follow the proof of Proposition 2.4 in [36. An alternative form and proof of the following equivalence can be found in Lemma D.1 of [16].

Lemma 2.11. Let $T>0, x, y \in G, p \in(1, \infty)$, and $C \in(0, \infty]$. Then

$$
\left(P_{T} f\right)^{p}(x) \leq C P_{T} f^{p}(y), \quad \text { for all } f \in L^{\infty}(G) \text { with } f \geq 0,
$$

if and only if

$$
\left(\int_{G}\left[\frac{p_{T}(x, z)}{p_{T}(y, z)}\right]^{1 /(p-1)} p_{T}(x, z) d z\right)^{p-1} \leq C .
$$


Proof. Set $J_{x, y}(z)=\frac{p_{T}(x, z)}{p_{T}(y, z)}$ and $f_{n}:=\left(n \wedge J_{x, y}\right)^{1 /(p-1)}$ for $n \geq 1$. Then applying (2.10) to $f_{n}$ yields

$$
\begin{aligned}
\left(P_{T} f_{n}\right)^{p}(x) & \leq C P_{T} f_{n}^{p}(y)=C \int_{G}\left(n \wedge J_{x, y}(z)\right)^{p /(p-1)} p_{T}(y, z) d z \\
& \leq C \int_{G}\left(n \wedge \frac{p_{T}(x, z)}{p_{T}(y, z)}\right)^{1 /(p-1)} p_{T}(x, z) d z=C P_{T} f_{n}(x) .
\end{aligned}
$$

Thus,

$$
P_{T} J_{x, y}^{1 /(p-1)}(x)=\lim _{n \rightarrow \infty} P_{T} f_{n}(x) \leq C^{1 /(p-1)},
$$

which yields (2.11).

For the converse, we have by Hölder's inequality that

$$
\begin{aligned}
P_{T} f(x) & =\int_{G} f(z) \frac{p_{T}(x, z)}{p_{T}(y, z)} p_{T}(y, z) d z \\
& \leq\left(P_{T} f^{p}\right)^{1 / p}(y)\left(\int_{G}{\frac{p_{T}(x, z)}{p_{T}(y, z)}}^{p /(p-1)} p_{T}(y, z) d z\right)^{(p-1) / p} \\
& =\left(P_{T} f^{p}\right)^{1 / p}(y)\left(\int_{G}{\frac{p_{T}(x, z)^{1 /(p-1)}}{p_{T}(y, z)}}^{(p-1) / p} p_{T}(x, z) d z\right)^{(p)} \\
& \leq\left(P_{T} f^{p}\right)^{1 / p}(y) C^{1 / p},
\end{aligned}
$$

which completes the proof.

\section{INFINITE-DIMENSIONAL HEISENBERG-LIKE GROUPS}

In this section, we review the definitions for infinite-dimensional Heisenberglike groups, which are infinite-dimensional Lie groups based on an abstract Wiener space. Much of the material is this section also appears in [19.

3.1. Abstract Wiener spaces. For the reader's convenience, we summarize several well-known properties of Gaussian measures and abstract Wiener spaces that are required for the sequel. These results, as well as more details on abstract Wiener spaces and some particular examples, may be found in [10, 25.

Suppose that $W$ is a real separable Banach space and $\mathcal{B}_{W}$ is the Borel $\sigma$-algebra on $W$.

Definition 3.1. A measure $\mu$ on $\left(W, \mathcal{B}_{W}\right)$ is called a (mean zero, non-degenerate) Gaussian measure provided that its characteristic functional is given by

$$
\hat{\mu}(u):=\int_{W} e^{i u(x)} d \mu(x)=e^{-\frac{1}{2} q(u, u)}, \quad \text { for all } u \in W^{*},
$$

for $q=q_{\mu}: W^{*} \times W^{*} \rightarrow \mathbb{R}$ a symmetric, positive definite quadratic form. That is, $q$ is a real inner product on $W^{*}$.

Lemma 3.2. If $u, v \in W^{*}$, then

$$
\int_{W} u(w) v(w) d \mu(w)=q(u, v) .
$$


Proof. Let $u_{*} \mu:=\mu \circ u^{-1}$ denote the measure on $\mathbb{R}$ which is the push forward of $\mu$ under $u$. Then by equation (3.1) $u_{*} \mu$ is normal with mean 0 and variance $q(u, u)$. Thus,

$$
\int_{W} u^{2}(w) d \mu(w)=q(u, u)
$$

Polarizing this identity gives the desired result.

A proof of the following standard theorem may be found for example in Appendix A of [15].

Theorem 3.3. Let $\mu$ be a Gaussian measure on a real separable Banach space $W$. For $p \in[1, \infty)$, let

$$
C_{p}:=\int_{W}\|w\|_{W}^{p} d \mu(w)
$$

For $w \in W$, let

$$
\|w\|_{H}:=\sup _{u \in W^{*} \backslash\{0\}} \frac{|u(w)|}{\sqrt{q(u, u)}}
$$

and define the Cameron-Martin subspace $H \subset W$ by

$$
H:=\left\{h \in W:\|h\|_{H}<\infty\right\} .
$$

Then

(1) For all $p \in[1, \infty), C_{p}<\infty$.

(2) $H$ is a dense subspace of $W$.

(3) There exists a unique inner product $\langle\cdot, \cdot\rangle_{H}$ on $H$ such that $\|h\|_{H}^{2}=\langle h, h\rangle_{H}$ for all $h \in H$, and $H$ is a separable Hilbert space with respect to this inner product.

(4) For any $h \in H,\|h\|_{W} \leq \sqrt{C_{2}}\|h\|_{H}$.

(5) If $\left\{e_{j}\right\}_{j=1}^{\infty}$ is an orthonormal basis for $H$, then for any $u, v \in H^{*}$,

$$
q(u, v)=\langle u, v\rangle_{H^{*}}=\sum_{j=1}^{\infty} u\left(e_{j}\right) v\left(e_{j}\right) .
$$

It follows from item (4) that any $u \in W^{*}$ restricted to $H$ is in $H^{*}$. Therefore, by item (5) and Lemma 3.2 .

$$
\int_{W} u^{2}(w) d \mu(w)=q(u, u)=\|u\|_{H^{*}}^{2}=\sum_{j=1}^{\infty}\left|u\left(e_{j}\right)\right|^{2} .
$$

More generally we have the following lemma.

Lemma 3.4. Let $K$ be a real Hilbert space and $\varphi: W \rightarrow K$ be a linear map. Then

$$
\|\varphi\|_{H^{*} \otimes K}^{2}=\sum_{j=1}^{\infty}\left\|\varphi\left(e_{j}\right)\right\|_{K}^{2}=\int_{W}\|\varphi(w)\|_{K}^{2} d \mu(w) .
$$


Proof. Let $\left\{f_{\ell}\right\}_{\ell=1}^{\operatorname{dim}(K)}$ be an orthonormal basis of $K$. Then, by equation (3.3),

$$
\begin{aligned}
\int_{W}\|\varphi(w)\|_{K}^{2} d \mu(w) & =\int_{W} \sum_{\ell=1}^{\operatorname{dim}(K)}\left|\left\langle\varphi(w), f_{\ell}\right\rangle_{K}\right|^{2} d \mu(w) \\
& =\sum_{\ell=1}^{\operatorname{dim}(K)}\left\|\left\langle\varphi(\cdot), f_{\ell}\right\rangle_{K}\right\|_{H^{*}}^{2}=\sum_{\ell=1}^{\operatorname{dim}(K)} \sum_{j=1}^{\infty}\left|\left\langle\varphi\left(e_{j}\right), f_{\ell}\right\rangle_{K}\right|^{2} \\
& =\sum_{j=1}^{\infty}\left\|\varphi\left(e_{j}\right)\right\|_{K}^{2}=\|\varphi\|_{H^{*} \otimes K}^{2}
\end{aligned}
$$

This leads to the following facts for linear maps on $W$. First we set the same notation.

Notation 3.5. Let $K$ be a real Hilbert space, and suppose $\alpha: H^{\otimes m} \rightarrow K$ is a multi-linear map. Then the Hilbert-Schmidt norm of $\alpha$ is defined by

$$
\begin{aligned}
\|\alpha\|_{2}^{2}:=\|\alpha\|_{\left(H^{*}\right) \otimes m \otimes K}^{2} & =\sum_{j_{1}, \ldots, j_{m}=1}^{\infty}\left\|\alpha\left(e_{j_{1}}, \ldots, e_{j_{m}}\right)\right\|_{K}^{2} \\
& =\sum_{j_{1}, \ldots, j_{m}=1}^{\infty} \sum_{\ell=1}^{\operatorname{dim}(K)}\left\langle\alpha\left(e_{j_{1}}, \ldots, e_{j_{m}}\right), f_{\ell}\right\rangle_{K}^{2},
\end{aligned}
$$

where $\left\{e_{j}\right\}_{j=1}^{\infty}$ and $\left\{f_{\ell}\right\}_{\ell=1}^{\operatorname{dim}(K)}$ are orthonormal bases of $H$ and $K$, respectively.

One may verify directly that these norms are independent of the chosen bases.

Lemma 3.6. Suppose $K$ is a Hilbert space and $\varphi: W \rightarrow K$ is a continuous linear map. Then $\varphi: H \rightarrow K$ is Hilbert-Schmidt, that is, $\|\varphi\|_{2}<\infty$.

Proof. By Lemma 3.4

$$
\begin{aligned}
\|\varphi\|_{2}^{2}=\|\varphi\|_{H^{*} \otimes K}^{2} & =\int_{W}\|\varphi(w)\|_{K}^{2} d \mu(w) \\
& \leq\|\varphi\|_{0}^{2} \int_{W}\|w\|_{W}^{2} d \mu(w)=C_{2}\|\varphi\|_{0}^{2},
\end{aligned}
$$

where $C_{2}<\infty$ is as defined in $(3.2)$ and

$$
\|\varphi\|_{0}:=\sup \left\{\varphi(w)\left\|_{K}:\right\| w \|_{W}=1\right\}<\infty
$$

by the continuity of $\varphi$.

Similarly, we may prove the following.

Lemma 3.7. Suppose $K$ is a Hilbert space and $\rho: W \times W \rightarrow K$ is a continuous bilinear map. Then $\rho: H \times H \rightarrow K$ is Hilbert-Schmidt.

Proof. Note first that, for each $w \in W, \varphi=\rho(w, \cdot)$ is a continuous linear operator and thus, by the proof of Lemma 3.6 .

$$
\|\rho(w, \cdot)\|_{2}^{2}=\|\rho(w, \cdot)\|_{H^{*} \otimes K} \leq C_{2}\|\rho(w, \cdot)\|_{0}^{2} \leq C_{2}\|\rho\|_{0}\|w\|_{W}^{2},
$$

where

$$
\|\rho\|_{0}:=\sup \left\{\rho\left(w, w^{\prime}\right)\left\|_{K}:\right\| w\left\|_{W}=\right\| w^{\prime} \|_{W}=1\right\}<\infty .
$$


Then viewing $w \mapsto \rho(w, \cdot)$ as a continuous linear map from $W$ to the Hilbert space $H^{*} \otimes K$, Lemma 3.4 implies that

$$
\begin{aligned}
\|\rho\|_{2}^{2} & =\|h \mapsto \rho(h, \cdot)\|_{H^{*} \otimes\left(H^{*} \otimes K\right)}^{2}=\int_{W}\|\rho(w, \cdot)\|_{H^{*} \otimes K}^{2} d \mu(w) \\
& \leq \int_{W} C_{2}\|\rho\|_{0}^{2}\|w\|_{W}^{2} d \mu(w)=C_{2}^{2}\|\rho\|_{0}^{2}<\infty .
\end{aligned}
$$

3.2. Infinite-dimensional Heisenberg-like groups. We revisit the definition of the infinite-dimensional Heisenberg-like groups that were first considered in [15. Note that since we are interested in subelliptic heat kernel measures on these groups, there are some necessary modifications to the topology as was done in [19]. First we set the following notation which will hold for the rest of the paper.

Notation 3.8. Let $(W, H, \mu)$ be a real abstract Wiener space. Let $\mathbf{C}$ be a real Hilbert space with inner product $\langle\cdot, \cdot\rangle_{\mathbf{C}}$ and $\operatorname{dim}(\mathbf{C})=N<\infty$. Let $\omega: W \times W \rightarrow \mathbf{C}$ be a continuous skew-symmetric bilinear form on $W$. We will also trivially assume that $\omega$ is surjective (otherwise, we just restrict ourselves to a linear subspace of $\mathbf{C}$ ).

Definition 3.9. Let $\mathfrak{g}$ denote $W \times \mathbf{C}$ when thought of as a Lie algebra with the Lie bracket given by

$$
\left[\left(X_{1}, V_{1}\right),\left(X_{2}, V_{2}\right)\right]:=\left(0, \omega\left(X_{1}, X_{2}\right)\right) .
$$

Let $G$ denote $W \times \mathbf{C}$ when thought of as a group with multiplication given by

$$
g_{1} g_{2}:=g_{1}+g_{2}+\frac{1}{2}\left[g_{1}, g_{2}\right],
$$

where $g_{1}$ and $g_{2}$ are viewed as elements of $\mathfrak{g}$. For $g_{i}=\left(w_{i}, c_{i}\right)$, this may be written equivalently as

$$
\left(w_{1}, c_{1}\right) \cdot\left(w_{2}, c_{2}\right)=\left(w_{1}+w_{2}, c_{1}+c_{2}+\frac{1}{2} \omega\left(w_{1}, w_{2}\right)\right) .
$$

We will call $G$ constructed in this way a Heisenberg-like group.

It is easy to verify that, given this bracket and multiplication, $\mathfrak{g}$ is indeed a Lie algebra and $G$ is a group. Note that $g^{-1}=-g$ and the identity $e=(0,0)$.

Notation 3.10. Let $\mathfrak{g}_{C M}$ denote $H \times \mathbf{C}$ when thought of as a Lie subalgebra of $\mathfrak{g}$, and we will refer to $\mathfrak{g}_{C M}$ as the Cameron-Martin subalgebra of $\mathfrak{g}$. Similarly, let $G_{C M}$ denote $H \times \mathbf{C}$ when thought of as a subgroup of $G$, and we will refer to $G_{C M}$ as the Cameron-Martin subgroup of $G$.

We will equip $\mathfrak{g}=G$ with the homogeneous norm

$$
\|(w, c)\|_{\mathfrak{g}}:=\sqrt{\|w\|_{W}^{2}+\|c\|_{\mathbf{C}}},
$$

and analogously on $\mathfrak{g}_{C M}=G_{C M}$ we define

$$
\|(A, a)\|_{\mathfrak{g}_{C M}}:=\sqrt{\|A\|_{H}^{2}+\|a\|_{\mathbf{C}}} .
$$

One may easily see that $G$ and $G_{C M}$ are topological groups with respect to the topologies induced by the homogeneous norms; see for example Lemma 2.9 of [19]. 
Before proceeding, let us give the basic motivating examples for the construction of these infinite-dimensional Heisenberg-like groups. In what follows, if $X$ is a complex vector space, let $X_{\operatorname{Re}}$ denote $X$ thought of as a real vector space. If $\left(H,\langle\cdot, \cdot\rangle_{H}\right)$ is a complex Hilbert space, let $\langle\cdot, \cdot\rangle_{H_{\mathrm{Re}}}:=\operatorname{Re}\langle\cdot, \cdot\rangle_{H}$, in which case $\left(H_{\mathrm{Re}},\langle\cdot, \cdot\rangle_{H_{\mathrm{Re}}}\right)$ becomes a real Hilbert space.

Example 3.11 (Finite-dimensional Heisenberg group). Let $W=H=\left(\mathbb{C}^{n}\right)_{\operatorname{Re}} \cong$ $\mathbb{R}^{2 n}$ and $\mu$ be standard Gaussian measure on $\mathbb{R}^{2 n}$. Then $(W, H, \mu)$ is an abstract Wiener space. Let $\mathbf{C}=\mathbb{R}$ and $\omega(w, z):=\operatorname{Im}\langle w, z\rangle$, where $\langle w, z\rangle=w \cdot \bar{z}$ is the usual inner product on $\mathbb{C}^{n}$. Then $G=\mathbb{R}^{2 n} \times \mathbb{R}$, equipped with a group operation as defined in (3.5), is a finite-dimensional Heisenberg group.

Example 3.12 (Heisenberg group of a symplectic vector space). Let $(K,\langle\cdot, \cdot\rangle)$ be a complex Hilbert space and $Q$ be a strictly positive trace class operator on $K$. For $h, k \in K$, let $\langle h, k\rangle_{Q}:=\langle h, Q k\rangle$ and $\|h\|_{Q}:=\sqrt{\langle h, h\rangle_{Q}}$, and let $\left(K_{Q},\langle\cdot, \cdot\rangle_{Q}\right)$ denote the Hilbert space completion of $\left(K,\|\cdot\|_{Q}\right)$. Then $W=\left(K_{Q}\right)_{\operatorname{Re}}$ and $H=K_{\operatorname{Re}}$ determines an abstract Wiener space (see, for example, exercise 17 on p. 59 of [25]). Letting $\mathbf{C}=\mathbb{R}$ and

$$
\omega(w, z):=\operatorname{Im}\langle w, z\rangle_{Q},
$$

then $G=\left(K_{Q}\right)_{\operatorname{Re}} \times \mathbb{R}$, equipped with a group operation as defined in (3.5), is an infinite-dimensional Heisenberg-like group.

3.3. Finite-dimensional projection groups. The finite-dimensional projections of $G$ defined in this section will be important in the sequel. Note that the construction of these projections is quite natural in the sense that they come from the usual projections of the abstract Wiener space. However, the projections defined here are not group homomorphisms, which is a complicating factor in the analysis.

As usual, let $(W, H, \mu)$ denote an abstract Wiener space. Let $i: H \rightarrow W$ be the inclusion map, and $i^{*}: W^{*} \rightarrow H^{*}$ be its transpose so that $i^{*} \ell:=\ell \circ i$ for all $\ell \in W^{*}$. Also, let

$$
H_{*}:=\left\{h \in H:\langle\cdot, h\rangle_{H} \in \operatorname{Range}\left(i^{*}\right) \subset H^{*}\right\} .
$$

That is, for $h \in H, h \in H_{*}$ if and only if $\langle\cdot, h\rangle_{H} \in H^{*}$ extends to a continuous linear functional on $W$, which we will continue to denote by $\langle\cdot, h\rangle_{H}$. Because $H$ is a dense subspace of $W, i^{*}$ is injective and thus has a dense range. Since $H \ni h \mapsto\langle\cdot, h\rangle_{H} \in$ $H^{*}$ is a linear isometric isomorphism, it follows that $H_{*} \ni h \mapsto\langle\cdot, h\rangle_{H} \in W^{*}$ is a linear isomorphism also, and so $H_{*}$ is a dense subspace of $H$.

Suppose that $P: H \rightarrow H$ is a finite rank orthogonal projection such that $P H \subset$ $H_{*}$. Let $\left\{e_{j}\right\}_{j=1}^{n}$ be an orthonormal basis for $P H$. Then we may extend $P$ to a (unique) continuous operator from $W \rightarrow H$ (still denoted by $P$ ) by letting

$$
P w:=\sum_{j=1}^{n}\left\langle w, e_{j}\right\rangle_{H} e_{j}
$$

for all $w \in W$.

Notation 3.13. Let $\operatorname{Proj}(W)$ denote the collection of finite rank projections on $W$ such that

(1) $P W \subset H_{*}$,

(2) $\left.P\right|_{H}: H \rightarrow H$ is an orthogonal projection (that is, $P$ has the form given in equation (3.6) $)$, and 
(3) $P W$ is sufficiently large to satisfy Hörmander's condition (that is, $\{\omega(A, B)$ : $A, B \in P W\}=\mathbf{C})$.

For each $P \in \operatorname{Proj}(W)$, we define $G_{P}:=P W \times \mathbf{C} \subset H_{*} \times \mathbf{C}$ and a corresponding projection $\pi_{P}: G \rightarrow G_{P}$,

$$
\pi_{P}(w, x):=(P w, x) .
$$

We will also let $\mathfrak{g}_{P}=\operatorname{Lie}\left(G_{P}\right)=P W \times \mathbf{C}$. In the context of Section 2, note that, for each $P \in \operatorname{Proj}(W), G_{P}$ is a finite-dimensional connected unimodular Lie group (in fact, $\mathfrak{g}_{P}$ is step 2 stratified) with $\mathcal{H}=P H$ and $\mathcal{V}=\mathbf{C}$.

3.4. Derivatives and differential forms on $G$. For $x \in G$, again let $L_{x}: G \rightarrow G$ and $R_{x}: G \rightarrow G$ denote left and right multiplication by $x$, respectively. As $G$ is a vector space, to each $x \in G$ we can associate the tangent space $T_{x} G$ to $G$ at $x$, which is naturally isomorphic to $G$.

Notation 3.14 (Linear and group derivatives). Let $f: G \rightarrow \mathbb{C}$ denote a Fréchet smooth function for $G$ considered as a Banach space with respect to the norm

$$
|(w, c)|_{G}:=\sqrt{\|w\|_{W}^{2}+\|c\|_{\mathbf{C}}^{2}}
$$

Then, for $x \in G$, and $h, k \in \mathfrak{g}$, let

$$
f^{\prime}(x) h:=\partial_{h} f(x)=\left.\frac{d}{d t}\right|_{0} f(x+t h)
$$

and

$$
f^{\prime \prime}(x)(h \otimes k):=\partial_{h} \partial_{k} f(x) .
$$

For $v, x \in G$, let $v_{x} \in T_{x} G$ denote the tangent vector satisfying $v_{x} f=f^{\prime}(x) v$. If $x(t)$ is any smooth curve in $G$ such that $x(0)=x$ and $\dot{x}(0)=v$ (for example, $x(t)=x+t v)$, then

$$
L_{g *} v_{x}=\left.\frac{d}{d t}\right|_{0} g \cdot x(t) .
$$

In particular, for $x=e$ and $v_{e}=h \in \mathfrak{g}$, again we let $\tilde{h}(g):=L_{g *} h$ so that $\tilde{h}$ is the unique left invariant vector field on $G$ such that $\tilde{h}(e)=h$. As usual, we view $\tilde{h}$ as a first order differential operator acting on smooth functions by

$$
(\tilde{h} f)(x)=\left.\frac{d}{d t}\right|_{0} f(x \cdot \sigma(t)),
$$

where $\sigma(t)$ is a smooth curve in $G$ such that $\sigma(0)=e$ and $\dot{\sigma}(0)=h$ (for example, $\sigma(t)=t h)$.

Proposition 3.15. Let $f: G \rightarrow \mathbb{R}$ be a smooth function, $h=(A, a) \in \mathfrak{g}$ and $x=(w, c) \in G$. Then

$$
\widetilde{h}(x):=l_{x *} h=\left(A, a+\frac{1}{2} \omega(w, A)\right)_{x} \text { for all } x=(w, c) \in G
$$

and, in particular,

$$
\widetilde{(A, a)} f(x)=f^{\prime}(x)\left(A, a+\frac{1}{2} \omega(w, A)\right) .
$$

Furthermore, if $h=(A, a), k=(B, b)$, then

$$
(\tilde{h} \tilde{k} f-\tilde{k} \tilde{h} f)=\widetilde{[h, k]} f \text {. }
$$


That is, the Lie algebra structure on $\mathfrak{g}$ induced by the Lie algebra structure on the left invariant vector fields on $G$ is the same as the Lie algebra structure defined in equation (3.4).

Proof. Since $t h=t(A, a)$ is a curve in $G$ passing through the identity at $t=0$, we have

$$
\begin{aligned}
\widetilde{h}(x) & =\left.\frac{d}{d t}\right|_{0}[x \cdot(t h)]=\left.\frac{d}{d t}\right|_{0}[(w, c) \cdot t(A, a)] \\
& =\left.\frac{d}{d t}\right|_{0}\left[\left(w+t A, c+t a+\frac{t}{2} \omega(w, A)\right)\right] \\
& =\left(A, a+\frac{1}{2} \omega(w, A)\right) .
\end{aligned}
$$

So by the chain rule, $(\tilde{h} f)(x)=f^{\prime}(x) \tilde{h}(x)$, and hence

$$
\begin{aligned}
(\tilde{h} \tilde{k} f)(x) & =\left.\frac{d}{d t}\right|_{0}\left[f^{\prime}(x \cdot t h) \tilde{k}(x \cdot t h)\right] \\
& =f^{\prime \prime}(x)(\tilde{h}(x) \otimes \tilde{k}(x))+\left.f^{\prime}(x) \frac{d}{d t}\right|_{0} \tilde{k}(x \cdot t h),
\end{aligned}
$$

where

$$
\left.\frac{d}{d t}\right|_{0} \tilde{k}(x \cdot t h)=\left.\frac{d}{d t}\right|_{0}\left(B, a+\frac{1}{2} \omega(w+t A, B)\right)=\left(0, \frac{1}{2} \omega(A, B)\right) .
$$

Since $f^{\prime \prime}(x)$ is symmetric, it now follows by subtracting equation (3.9) from itself with $h$ and $k$ interchanged that

$$
(\tilde{h} \tilde{k} f-\tilde{k} \tilde{h} f)(x)=f^{\prime}(x)(0, \omega(A, B))=f^{\prime}(x)[h, k]=(\widetilde{[h, k]} f)(x),
$$

as desired.

Definition 3.16. A function $f: G \rightarrow \mathbb{C}$ is a (smooth) cylinder function if it may be written as $f=F \circ \pi_{P}$, for some $P \in \operatorname{Proj}(W)$ and (smooth) $F: G_{P} \rightarrow \mathbb{C}$.

The following lemma is Lemma 3.16 of [15. We reproduce the proof here for the reader's convenience.

Lemma 3.17. Suppose that $\ell: H \rightarrow \mathbf{C}$ is a continuous linear map. Then for any orthonormal basis $\left\{e_{j}\right\}_{j=1}^{\infty}$ of $H$ the series

$$
\sum_{j=1}^{\infty} \ell\left(e_{j}\right) \otimes \ell\left(e_{j}\right) \in \mathbf{C} \otimes \mathbf{C}
$$

and

$$
\sum_{j=1}^{\infty} \ell\left(e_{j}\right) \otimes e_{j} \in \mathbf{C} \otimes H
$$

are convergent and independent of the basis. 
Proof. First note that, since $\operatorname{dim}(\mathbf{C})=N<\infty, \ell$ is Hilbert-Schmidt since it is bounded, and thus, for $\left\{f_{i}\right\}_{i=1}^{N}$ an orthonormal basis of $\mathbf{C}$,

$$
\begin{aligned}
\sum_{j=1}^{\infty}\left\|\ell\left(e_{j}\right) \otimes \ell\left(e_{j}\right)\right\|_{\mathbf{C} \otimes \mathbf{C}} & =\sum_{j=1}^{\infty}\left\|\ell\left(e_{j}\right)\right\|_{\mathbf{C}}^{2} \\
& =\sum_{i=1}^{N} \sum_{j=1}^{\infty}\left\langle f_{i}, \ell\left(e_{j}\right)\right\rangle_{\mathbf{C}}^{2}=\sum_{i=1}^{N}\left\|\left\langle f_{i}, \ell(\cdot)\right\rangle\right\|_{H^{*}}^{2}<\infty .
\end{aligned}
$$

This also shows that the sum in equation (3.10) is absolutely convergent. Similarly, since $\left\{\ell\left(e_{j}\right) \otimes e_{j}\right\}_{j=1}^{\infty}$ is an orthogonal set in $\mathbf{C} \otimes H$ and

$$
\sum_{j=1}^{\infty}\left\|\ell\left(e_{j}\right) \otimes e_{j}\right\|_{\mathbf{C} \otimes H}^{2}=\sum_{j=1}^{\infty}\left\|\ell\left(e_{j}\right)\right\|_{\mathbf{C}}^{2}<\infty,
$$

the sum in equation (3.11) is convergent as well.

Now recall that if $H$ and $K$ are two real Hilbert spaces, then the Hilbert space tensor product $H \otimes K$ is unitarily equivalent to the space of Hilbert-Schmidt operators $H S(H, K)$ from $H$ to $K$. Under this identification, $h \otimes k \in H \otimes K$ corresponds to the operator (still denoted by $h \otimes k)$ in $H S(H, K)$ defined by

$$
H \ni h^{\prime} \mapsto(h \otimes k) h^{\prime}=\left\langle h, h^{\prime}\right\rangle_{H} k \in K .
$$

Using this identification we have that, for all $c \in \mathbf{C}$,

$$
\begin{aligned}
\left(\sum_{j=1}^{\infty} \ell\left(e_{j}\right) \otimes \ell\left(e_{j}\right)\right) c & =\sum_{j=1}^{\infty} \ell\left(e_{j}\right)\left\langle\ell\left(e_{j}\right), c\right\rangle_{\mathbf{C}}=\sum_{j=1}^{\infty} \ell\left(e_{j}\right)\left\langle e_{j}, \ell^{*} c\right\rangle_{\mathbf{C}} \\
& =\ell\left(\sum_{j=1}^{\infty}\left\langle e_{j}, \ell^{*} c\right\rangle_{\mathbf{C}} e_{j}\right)=\ell \ell^{*} c
\end{aligned}
$$

and

$$
\left(\sum_{j=1}^{\infty} \ell\left(e_{j}\right) \otimes e_{j}\right) c=\sum_{j=1}^{\infty} e_{j}\left\langle\ell\left(e_{j}\right), c\right\rangle_{\mathbf{C}}=\sum_{j=1}^{\infty} e_{j}\left\langle e_{j}, \ell^{*} c\right\rangle_{\mathbf{C}}=\ell^{*} c
$$

which clearly shows that equations (3.10) and(3.11) are basis-independent.

Notation 3.18. For $x=(w, c) \in G$, let $\gamma(x)$ and $\chi(x)$ be the elements of $\mathfrak{g}_{C M} \otimes \mathfrak{g}_{C M}$ defined by

$$
\begin{aligned}
& \gamma(x):=\sum_{j=1}^{\infty}\left(0, \omega\left(w, e_{j}\right)\right) \otimes\left(e_{j}, 0\right) \text { and } \\
& \chi(x):=\sum_{j=1}^{\infty}\left(0, \omega\left(w, e_{j}\right)\right) \otimes\left(0, \omega\left(w, e_{j}\right)\right),
\end{aligned}
$$

where $\left\{e_{j}\right\}_{j=1}^{\infty}$ is any orthonormal basis for $H$. Both $\gamma$ and $\chi$ are well defined by Lemma 3.17 taking $\ell=\omega(x, \cdot)$.

The following proposition is proved in Proposition 3.29 of [15], although the statement given there is for the elliptic case. Again, we reproduce the short proof here for the reader's convenience. 
Proposition 3.19. Let $\left\{e_{j}\right\}_{j=1}^{\infty}$ be an orthonormal basis for $H$. Then, for any smooth cylinder function $f: G \rightarrow \mathbb{R}$,

$$
L f(x):=\sum_{j=1}^{\infty}\left[{\widetilde{\left(e_{j}, 0\right)}}^{2} f\right](x)
$$

is well defined and independent of basis. In particular, if $f=F \circ \pi_{P}, x=(w, c) \in G$, $\partial_{h}$ is as in Notation 3.14 for all $h \in \mathfrak{g}_{C M}$, and

$$
\Delta_{H} f(x):=\sum_{j=1}^{\infty} \partial_{\left(e_{j}, 0\right)}^{2} f(x)=\left(\Delta_{P H} F\right)(P w, c),
$$

then

$$
L f(x)=\left(\Delta_{H} f\right)(x)+f^{\prime \prime}(x)\left(\gamma(x)+\frac{1}{4} \chi(x)\right),
$$

where $f^{\prime \prime}$ is as defined in Notation 3.14 and $\gamma$ and $\chi$ are as defined in Notation 3.18 .

Proof. Recall from equation (3.7) that

$$
\widetilde{\left(e_{j}, 0\right)} f(x)=f^{\prime}(x)\left(e_{j}, \frac{1}{2} \omega\left(w, e_{j}\right)\right)
$$

where $f^{\prime}$ is also as defined in Notation 3.14 . Applying $\widetilde{\left(e_{j}, 0\right)}$ to both sides of this equation then gives

$$
\begin{aligned}
{\widetilde{\left(e_{j}, 0\right)}}^{2} f(x)= & f^{\prime \prime}(x)\left(\left(e_{j}, \frac{1}{2} \omega\left(w, e_{j}\right)\right) \otimes\left(e_{j}, \frac{1}{2} \omega\left(w, e_{j}\right)\right)\right) \\
= & f^{\prime \prime}(x)\left(\left(e_{j}, 0\right) \otimes\left(e_{j}, 0\right)\right)+f^{\prime \prime}(x)\left(\left(0, \omega\left(w, e_{j}\right)\right) \otimes\left(e_{j}, 0\right)\right) \\
& +\frac{1}{4} f^{\prime \prime}(x)\left(\left(0, \omega\left(w, e_{j}\right)\right) \otimes\left(0, \omega\left(w, e_{j}\right)\right)\right),
\end{aligned}
$$

wherein we have used that

$$
\partial_{e_{j}} \omega\left(\cdot, e_{j}\right)=\omega\left(e_{j}, e_{j}\right)=0
$$

and that $f^{\prime \prime}(x)$ is symmetric. Summing on $j$ then shows that

$$
\begin{aligned}
\sum_{j=1}^{\infty}\left[{\widetilde{\left(e_{j}, 0\right)}}^{2} f\right](x) & =\sum_{j=1}^{\infty} f^{\prime \prime}(x)\left(\left(e_{j}, 0\right) \otimes\left(e_{j}, 0\right)\right)+f^{\prime \prime}(x)\left(\gamma(x)+\frac{1}{4} \chi(x)\right) \\
& =\sum_{j=1}^{\infty} \partial_{\left(e_{j}, 0\right)}^{2} f(x)+f^{\prime \prime}(x)\left(\gamma(x)+\frac{1}{4} \chi(x)\right),
\end{aligned}
$$

which verifies equation (3.12) and thus shows that $L f$ is independent of the choice of an orthonormal basis for $H$.

Similarly, we may prove the following proposition.

Proposition 3.20. Let $\left\{e_{j}\right\}_{j=1}^{\infty}$ be an orthonormal basis for $H$. Then, for any smooth cylinder functions $f, g: G \rightarrow \mathbb{R}$,

$$
\Gamma(f, g)(x):=\sum_{j=1}^{\infty}\left(\widetilde{\left(e_{j}, 0\right)} f\right)(x)\left(\widetilde{\left(e_{j}, 0\right)} g\right)(x)
$$


is well defined and independent of basis. In particular, if $f=F \circ \pi_{P}$ and $g=G \circ \pi_{Q}$ for $P, Q \in \operatorname{Proj}(W), x=(w, c) \in G, \partial_{h}$ is as in Notation 3.14 for all $h \in \mathfrak{g}_{C M}$, and

$$
\nabla_{H} f(x):=\sum_{j=1}^{\infty}\left(\partial_{\left(e_{j}, 0\right)} f(x)\right) e_{j}=\nabla_{P H} F(P w, c),
$$

then

$$
\begin{aligned}
\Gamma(f, g)(x)=\left\langle\nabla_{H} f(x), \nabla_{H} g(x)\right\rangle_{H}+ & \frac{1}{4}\left(f^{\prime}(x) \otimes g^{\prime}(x)\right) \chi(x) \\
& +\frac{1}{2}\left(f^{\prime}(x) \otimes g^{\prime}(x)+g^{\prime}(x) \otimes f^{\prime}(x)\right) \gamma(x),
\end{aligned}
$$

where $f^{\prime}$ is as defined in Notation 3.14 and $\gamma$ and $\chi$ are as defined in Notation 3.18 .

Proof. Again recall from equation (3.7) that

$$
\begin{aligned}
\widetilde{\left(e_{j}, 0\right)} f(x) & =f^{\prime}(x)\left(e_{j}, \frac{1}{2} \omega\left(w, e_{j}\right)\right)=f^{\prime}(x)\left(\left(e_{j}, 0\right)+\frac{1}{2}\left(0, \omega\left(w, e_{j}\right)\right)\right) \\
& =\partial_{\left(e_{j}, 0\right)} f(x)+\frac{1}{2} f^{\prime}(x)\left(0, \omega\left(w, e_{j}\right)\right) .
\end{aligned}
$$

Thus,

$$
\begin{aligned}
& \Gamma(f, g)(x)=\sum_{j=1}^{\infty}\left\{\partial_{\left(e_{j}, 0\right)} f(x) \partial_{\left(e_{j}, 0\right)} g(x)+\frac{1}{2} f^{\prime}(x)\left(0, e_{j}\right) g^{\prime}(x)\left(0, \omega\left(w, e_{j}\right)\right)\right. \\
& \left.+\frac{1}{2} f^{\prime}(x)\left(0, \omega\left(w, e_{j}\right)\right) g^{\prime}(x)\left(0, e_{j}\right)+\frac{1}{4} f^{\prime}(x)\left(0, \omega\left(w, e_{j}\right)\right) g^{\prime}(x)\left(0, \omega\left(w, e_{j}\right)\right)\right\} .
\end{aligned}
$$

Note for example that

$$
\begin{aligned}
\sum_{j=1}^{\infty} f^{\prime}(x)(0, \omega & \left.\left(w, e_{j}\right)\right) g^{\prime}(x)\left(e_{j}, 0\right) \\
& =\sum_{j=1}^{\infty}\left\langle f^{\prime}(x) \otimes g^{\prime}(x),\left(0, \omega\left(w, e_{j}\right)\right) \otimes\left(e_{j}, 0\right)\right\rangle \\
& =\left\langle f^{\prime}(x) \otimes g^{\prime}(x), \sum_{j=1}^{\infty}\left(0, \omega\left(w, e_{j}\right)\right) \otimes\left(e_{j}, 0\right)\right\rangle \\
& =\left\langle f^{\prime}(x) \otimes g^{\prime}(x), \gamma(x)\right\rangle .
\end{aligned}
$$

Similarly,

$$
\begin{aligned}
\sum_{j=1}^{\infty} f^{\prime}(x)\left(0, \omega\left(w, e_{j}\right)\right) & g^{\prime}(x)\left(0, \omega\left(w, e_{j}\right)\right) \\
& =\left\langle f^{\prime}(x) \otimes g^{\prime}(x), \sum_{j=1}^{\infty}\left(0, \omega\left(w, e_{j}\right)\right) \otimes\left(0, \omega\left(w, e_{j}\right)\right)\right\rangle \\
& =\left\langle f^{\prime}(x) \otimes g^{\prime}(x), \chi(x)\right\rangle .
\end{aligned}
$$


Thus, along with $L$ and $\Gamma$, we are able to consider the following differential forms which are well defined for smooth cylinder functions $f, g$ on $G$ :

$$
\begin{aligned}
\Gamma_{2}(f, g) & :=\frac{1}{2}(L \Gamma(f, g)-\Gamma(f, L g)-\Gamma(g, L f)), \\
\Gamma^{Z}(f, g) & :=\sum_{\ell=1}^{N}\left(\widetilde{\left(0, f_{\ell}\right)} f\right)\left(\widetilde{\left(0, f_{\ell}\right)}\right), \text { and } \\
\Gamma_{2}^{Z}(f, g): & =\frac{1}{2}\left(L \Gamma^{Z}(f, g)-\Gamma^{Z}(f, L g)-\Gamma^{Z}(g, L f)\right) .
\end{aligned}
$$

Of course, for the finite-dimensional groups $G_{P}$ we may define the same forms for $f, g \in C^{\infty}\left(G_{P}\right)$ as were done for more general finite-dimensional groups in Section 2. These will be denoted by $L_{P}, \Gamma_{P}, \Gamma_{2, P}$, and $\Gamma_{2, P}^{Z}$. In particular, if $\left\{e_{i}\right\}_{i=1}^{n}$ is an orthonormal basis of $P H$, then

$$
L_{P} f=\sum_{j=1}^{n}{\widetilde{\left(e_{j}, 0\right)}}^{2} f \quad \text { and } \quad \Gamma_{P}(f, g)=\sum_{j=1}^{n}\left(\widetilde{\left(e_{j}, 0\right) f}\right)\left(\widetilde{\left(e_{j}, 0\right) g}\right) .
$$

3.5. Distances on $G_{C M}$. We define the sub-Riemannian distance on $G_{C M}$ analogously to how it was done in finite dimensions in Section 2 . We recall its relevant properties, including the fact that the topology induced by this metric is equivalent to the topology induced by $\|\cdot\|_{\mathfrak{g}_{C M}}$.

Notation 3.21 (Horizontal distance on $G_{C M}$ ). (1) For $x=(A, a) \in G_{C M}$, let

$$
|x|_{\mathfrak{g}_{C M}}^{2}:=\|A\|_{H}^{2}+\|a\|_{\mathbf{C}}^{2} .
$$

The length of a $C^{1}$-path $\sigma:[a, b] \rightarrow G_{C M}$ is defined as

$$
\ell(\sigma):=\int_{a}^{b}\left|L_{\sigma^{-1}(s) *} \dot{\sigma}(s)\right|_{\mathfrak{g}_{C M}} d s .
$$

(2) A $C^{1}$-path $\sigma:[a, b] \rightarrow G_{C M}$ is horizontal if $L_{\sigma(t)-{ }_{*}} \dot{\sigma}(t) \in H \times\{0\}$ for a.e. $t$. Let $C_{C M}^{1, h}$ denote the set of horizontal paths $\sigma:[0,1] \rightarrow G_{C M}$.

(3) The horizontal distance between $x, y \in G_{C M}$ is defined by

$$
d(x, y):=\inf \left\{\ell(\sigma): \sigma \in C_{C M}^{1, h} \text { such that } \sigma(0)=x \text { and } \sigma(1)=y\right\} .
$$

The horizontal distance is defined analogously on $G_{P}$ and will be denoted by $d_{P}$. In particular, for a sequence $\left\{P_{n}\right\}_{n=1}^{\infty} \subset \operatorname{Proj}(W)$, we will let $G_{n}:=G_{P_{n}}$ and $d_{n}:=d_{P_{n}}$.

Remark 3.22. Note that if $\sigma(t)=(A(t), a(t))$ is a horizontal path, then

$$
L_{\sigma(t)^{-1} *} \dot{\sigma}(t)=\left(\dot{A}(t), \dot{a}(t)-\frac{1}{2} \omega(A(t), \dot{A}(t))\right) \in H \times\{0\}
$$

implies that $\sigma$ must satisfy

$$
a(t)=a(0)+\frac{1}{2} \int_{0}^{t} \omega(A(s), \dot{A}(s)) d s,
$$

and the length of $\sigma$ is given by

$$
\ell(\sigma)=\int_{0}^{1}\left|L_{\sigma^{-1}(s) *} \dot{\sigma}(s)\right|_{\mathfrak{g}_{C M}} d s=\int_{0}^{1}\|\dot{A}(s)\|_{H} d s .
$$


The following proposition is Propositions 2.17 and 2.18 of [19. We refer the reader to that paper for the proof.

Proposition 3.23. If $\{\omega(A, B): A, B \in H\}=\mathbf{C}$, then there exist finite constants $K_{1}=K_{1}(\omega)$ and $K_{2}=K_{2}(N, \omega)$ such that

$$
K_{1}\left(\|A\|_{H}+\sqrt{\|a\|_{\mathbf{C}}}\right) \leq d(e,(A, a)) \leq K_{2}\left(\|A\|_{H}+\sqrt{\|a\|_{\mathbf{C}}}\right),
$$

for all $(A, a) \in \mathfrak{g}_{C M}$. In particular, this is sufficient to imply that the topologies induced by $d$ and $\|\cdot\|_{\mathfrak{g}_{C M}}$ are equivalent.

Remark 3.24. The equivalence of the homogeneous norm and horizontal distance topologies is a standard result in finite dimensions. However, the usual proof of this result relies on compactness arguments that must be avoided in infinite dimensions. Thus, the proof for Proposition 3.23 included in [19] necessarily relies on different methods particular to the structure of the present groups. Using these methods, we are currently unable to remove the dependence on $N=\operatorname{dim}(\mathbf{C})$ from the coefficient in the upper bound. The reader is referred to [19] for further details.

Lemma 3.25. Fix $P_{0} \in \operatorname{Proj}(W)$ and let $x \in G_{0}=G_{P_{0}}$. Let $\left\{P_{n}\right\}_{n=1}^{\infty} \subset \operatorname{Proj}(W)$ such that $P_{0} H \subset P_{n} H$ for all $n$ and $\left.P_{n}\right|_{H} \uparrow I_{H}$. Then

$$
d_{n}(e, x) \rightarrow d(e, x) \text {, as } n \rightarrow \infty,
$$

where $d_{n}$ is the horizontal distance on $G_{n}=G_{P_{n}}$ as in Notation 3.21

Proof. First, it is clear that, for any $n$ and $x, y \in G_{n}, d_{n}(x, y) \geq d(x, y)$. In particular, if $x, y \in G_{m}$ for some $m$, then $x, y \in G_{n}$ for all $n \geq m$ and $d_{n}(x, y)$ is decreasing as $n \rightarrow \infty$. Now let $x=(w, c) \in G_{0}$, and consider an arbitrary horizontal $C^{1}$-path $\sigma:[0,1] \rightarrow G_{C M}$ such that $\sigma(0)=e$ and $\sigma(1)=g$. Recall that, by Remark 3.22, $\sigma$ must have the form

$$
\sigma(t)=\left(A(t), \frac{1}{2} \int_{0}^{t} \omega(A(s), \dot{A}(s)) d s\right) .
$$

For $n \in \mathbb{N}$, consider the "projected" horizontal paths $\sigma_{n}:[0,1] \rightarrow G_{n}$ given by

$$
\sigma_{n}(t)=\left(A_{n}(t), a_{n}(t)\right):=\left(P_{n} A(t), \frac{1}{2} \int_{0}^{t} \omega\left(P_{n} A(s), P_{n} \dot{A}(s)\right) d s\right) .
$$

Note that $A_{n}(1)=P_{n} A(1)=P_{n} w=w$, and let

$$
\varepsilon_{n}:=c-a_{n}(1)=c-\frac{1}{2} \int_{0}^{1} \omega\left(P_{n} A(s), P_{n} \dot{A}(s)\right) d s \in \mathbf{C} .
$$

Then

$$
d_{n}(e, x)=d_{n}(e,(w, c))=d_{n}\left(e,\left(w, a_{n}(1)+\varepsilon_{n}\right)\right)=d_{n}\left(e,\left(w, a_{n}(1)\right) \cdot\left(0, \varepsilon_{n}\right)\right) .
$$

Now, for any left-invariant metric $d$, we have that

$$
d(e, x y) \leq d(e, x)+d(x, x y)=d(e, x)+d(e, y) .
$$

Thus,

$$
d_{n}(e, x) \leq d_{n}\left(e,\left(w, a_{n}(1)\right)\right)+d_{n}\left(e,\left(0, \varepsilon_{n}\right)\right) \leq \ell\left(\sigma_{n}\right)+C \sqrt{\left\|\varepsilon_{n}\right\|_{\mathbf{C}}},
$$

where the second inequality holds by (3.13) with constant $C=C(N, \omega)$ not depending on $n$. (Of course, the estimate (3.13) is stated for the horizontal distance $d$ on $G_{C M}$ and not $d_{n}$ on $G_{n}$. However, the proof of (3.13) in [19] shows that the same estimate holds for each $d_{P}$ with common coefficients $K_{1}$ and $K_{2}$ for all 
sufficiently large $P \in \operatorname{Proj}(W)$. For the sake of brevity, we do not include that proof here, but, roughly, the dependence on $N$ of the coefficient $K_{2}=K_{2}(N, \omega)$ appearing in Proposition 3.23 is determined by a choice of $\left\{A_{\ell}, B_{\ell}\right\}_{\ell=1}^{N} \subset H$ such that $\left\{\omega\left(A_{\ell}, B_{\ell}\right)\right\}_{\ell=1}^{N}$ is a basis of $\mathbf{C}$. By choosing $\left\{A_{\ell}, B_{\ell}\right\}_{\ell=1}^{N} \subset P_{0} H \subset P_{n} H$, one may find a constant $K_{2}$ independent of $n$ so that (3.13) holds with $d$ replaced by $d_{n}$. See the proof of Proposition 2.17 of [19] for complete details.)

Now, for any $k \geq n$, it is clear that $\ell\left(\sigma_{n}\right) \leq \ell\left(\sigma_{k}\right)$, since

$$
\ell\left(\sigma_{n}\right)=\int_{0}^{1}\left\|P_{n} \dot{A}(s)\right\|_{H} d s=\int_{0}^{1} \sqrt{\sum_{j=1}^{n}\left|\left\langle\dot{A}(s), e_{j}\right\rangle_{H}\right|^{2}} d s
$$

where $\left\{e_{j}\right\}_{j=1}^{n}$ is an orthonormal basis of $P_{n} H$. Thus, for all $k \geq n$,

$$
d_{n}(e, x) \leq \ell\left(\sigma_{k}\right)+C \sqrt{\left\|\varepsilon_{n}\right\|_{\mathbf{C}}}
$$

Dominated convergence implies that

$$
\lim _{k \rightarrow \infty} \ell\left(\sigma_{k}\right)=\lim _{k \rightarrow \infty} \int_{0}^{1}\left\|P_{k} \dot{A}(s)\right\| d s=\int_{0}^{1}\|\dot{A}(s)\| d s=\ell(\sigma),
$$

and thus allowing $k \rightarrow \infty$ in (3.14) gives

$$
d_{n}(e, x) \leq \ell(\sigma)+C \sqrt{\left\|\varepsilon_{n}\right\|_{\mathbf{C}}}
$$

Now taking the infimum over all horizontal paths in $G_{C M}$ such that $\sigma(0)=e$ and $\sigma(1)=g$ implies that

$$
d_{n}(e, x) \leq d(e, x)+C \sqrt{\left\|\varepsilon_{n}\right\|_{\mathbf{C}}}
$$

One may also show via dominated convergence that

$$
\lim _{n \rightarrow \infty}\left\|\varepsilon_{n}\right\|_{\mathbf{C}}=\lim _{n \rightarrow \infty}\left\|\frac{1}{2} \int_{0}^{1} \omega(A(s), \dot{A}(s))-\omega\left(P_{n} A(s), P_{n} \dot{A}(s)\right) d s\right\|_{\mathbf{C}}=0 .
$$

Thus, given an arbitrary $\varepsilon>0$, for all sufficiently large $n$,

$$
d(e, x) \leq d_{n}(e, x) \leq d(e, x)+\varepsilon .
$$

\section{INFINITE-DIMENSIONAL COMPUTATIONS}

Now given the structure of the infinite-dimensional Heisenberg-like groups and their finite-dimensional projections defined in the previous section, we wish to consider estimates such as the ones discussed in Section 2. In the first subsection, we will show that the desired curvature-dimension estimate (2.2) and commutation formula (2.6) hold for the differential forms defined on $G$ and $G_{P}$. In the second section, we then record the reverse inequalities and Harnack estimates that follow as a result. 
4.1. Curvature-dimension bounds and commutation relations. In this section, $\left\{e_{i}\right\}_{i=1}^{\infty}$ and $\left\{f_{\ell}\right\}_{\ell=1}^{N}$ will denote orthonormal bases for $H$ and $\mathbf{C}$ respectively, where $N=\operatorname{dim}(\mathbf{C})$. Let $\|\omega\|_{2}$ denote the Hilbert-Schmidt norm of $\omega: H \times H \rightarrow \mathbf{C}$ as defined in Notation 3.5 That is,

$$
\|\omega\|_{2}^{2}:=\|\omega\|_{H^{*} \otimes H^{*} \otimes \mathbf{C}}^{2}:=\sum_{i, j=1}^{\infty}\left\|\omega\left(e_{i}, e_{j}\right)\right\|_{\mathbf{C}}^{2}=\sum_{i, j=1}^{\infty} \sum_{\ell=1}^{N}\left\langle\omega\left(e_{i}, e_{j}\right), f_{\ell}\right\rangle_{\mathbf{C}}^{2} .
$$

As $\omega$ is a continuous bilinear operator on $W$, Lemma 3.7 implies that $\omega$ is HilbertSchmidt, and so $\|\omega\|_{2}<\infty$.

For the rest of this section, we also fix $P \in \operatorname{Proj}(W)$ and let $\left\{e_{i}\right\}_{i=1}^{n}$ denote an orthonormal basis of $P H$. Let $\|\omega\|_{2, P}$ denote the Hilbert-Schmidt norm of $\omega$ restricted to $P H$, that is,

$$
\|\omega\|_{2, P}^{2}:=\sum_{i, j=1}^{n}\left\|\omega\left(e_{i}, e_{j}\right)\right\|_{\mathbf{C}}^{2}=\sum_{i, j=1}^{n} \sum_{\ell=1}^{N}\left\langle\omega\left(e_{i}, e_{j}\right), f_{\ell}\right\rangle_{\mathbf{C}}^{2} .
$$

We will also let

$$
\begin{aligned}
\rho_{2} & :=\inf \left\{\sum_{i, j=1}^{\infty}\left(\sum_{\ell=1}^{N}\left\langle\omega\left(e_{i}, e_{j}\right), f_{\ell}\right\rangle_{\mathbf{C}} x_{\ell}\right)^{2}: \sum_{\ell=1}^{N} x_{\ell}^{2}=1\right\} \text { and } \\
\rho_{2, P} & :=\inf \left\{\sum_{i, j=1}^{n}\left(\sum_{\ell=1}^{N}\left\langle\omega\left(e_{i}, e_{j}\right), f_{\ell}\right\rangle_{\mathbf{C}} x_{\ell}\right)^{2}: \sum_{\ell=1}^{N} x_{\ell}^{2}=1\right\} .
\end{aligned}
$$

Remark 4.1. Note first that, given that $[P W, P W]=\mathbf{C}$, it must be that $\rho_{2, P}>0$. One way to see this is to let $F: \mathbb{R}^{N} \rightarrow \mathbb{R}$ be given by

$$
F(x)=F\left(x_{1}, \ldots, x_{N}\right):=\sum_{i, j=1}^{n}\left(\sum_{\ell=1}^{N}\left\langle\omega\left(e_{i}, e_{j}\right), f_{\ell}\right\rangle_{\mathbf{C}} x_{\ell}\right)^{2} .
$$

Then $F$ is a continuous function on $\mathbb{R}^{N}$ and so attains its minimum on the compact set $\|x\|=1$. Thus, if $\rho_{2, P}=\inf _{\|x\|=1} F(x)=\min _{\|x\|=1} F(x)=0$, there exists $x \in \mathbb{R}^{N}$ such that $\omega\left(e_{i}, e_{j}\right) \cdot x=0$ for all $i, j$. That is, $x$ is simultaneously orthogonal to all $\omega\left(e_{i}, e_{j}\right)$ and so $\left\{\omega\left(e_{i}, e_{j}\right)\right\}_{i, j=1}^{n}$ cannot $\operatorname{span} \mathbb{R}^{N} \cong \mathbf{C}$.

One may also see that $\rho_{2, P} \leq\|\omega\|_{2, P}$ and $\rho_{2} \leq\|\omega\|_{2}$. Furthermore, let $\left\{P_{n}\right\}_{n=1}^{\infty} \subset$ $\operatorname{Proj}(W)$ be an increasing sequence of projections such that $\left.P_{n}\right|_{H} \uparrow I_{H}$. Let $\Lambda_{n}$ be an increasing sequence of orthonormal bases for the $P_{n}$ and define $F_{n}$ analogously to (4.1) for each $P_{n}$. Then, for each $x$, the function $F_{n}(x)$ is increasing in $n$, and therefore $\rho_{2, P_{n}}$ is an increasing sequence. This, together with the fact that $\rho_{2, P_{n}} \leq \rho_{2}$ for all $n$, implies that $\rho_{2, P_{n}}$ must converge. In particular, it is clear that $\rho_{2, P_{n}} \uparrow \rho_{2}$. It is also clear that $\|\omega\|_{2, P_{n}} \uparrow\|\omega\|_{2}$.

We will need the following computational lemmas.

Lemma 4.2. For any smooth cylinder function $f$,

$$
\left.\rho_{2} \Gamma^{Z}(f) \leq \sum_{i, j=1}^{\infty}\left(\left(0, \widetilde{\omega\left(e_{i}, e_{j}\right.}\right)\right) f\right)^{2} \leq\|\omega\|_{2}^{2} \Gamma^{Z}(f) .
$$


Similarly, for any $f \in C^{\infty}\left(G_{P}\right)$,

$$
\left.\rho_{2, P} \Gamma^{Z}(f) \leq \sum_{i, j=1}^{n}\left(\left(0, \widetilde{\omega\left(e_{i}, e_{j}\right.}\right)\right) f\right)^{2} \leq\|\omega\|_{2, P}^{2} \Gamma^{Z}(f) .
$$

Proof. We will prove only the first set of inequalities, as the second proof is obviously parallel. The upper bound follows from the Cauchy-Schwarz inequality, since

$$
\begin{aligned}
& \left.\sum_{i, j=1}^{\infty}\left(\left(0, \widetilde{\omega\left(e_{i}, e_{j}\right.}\right)\right) f\right)^{2}=\sum_{i, j=1}^{\infty}\left(\sum_{\ell=1}^{N}\left\langle\omega\left(e_{i}, e_{j}\right), f_{\ell}\right\rangle_{\mathbf{C}} \widetilde{\left(0, f_{\ell}\right)} f\right)^{2} \\
& \quad \leq \sum_{i, j=1}^{\infty}\left(\sum_{\ell=1}^{N}\left\langle\omega\left(e_{i}, e_{j}\right), f_{\ell}\right\rangle_{\mathbf{C}}^{2}\right)\left(\sum_{\ell=1}^{N}\left(\widetilde{\left(0, f_{\ell}\right)} f\right)^{2}\right)=\|\omega\|_{2}^{2} \Gamma^{Z}(f) .
\end{aligned}
$$

To see the lower bound, simply note that

$$
\left.\sum_{i, j=1}^{\infty}\left(\left(0, \widetilde{\omega\left(e_{i}, e_{j}\right.}\right)\right) f\right)^{2}=\sum_{i, j=1}^{\infty}\left(\sum_{\ell=1}^{N}\left\langle\omega\left(e_{i}, e_{j}\right), f_{\ell}\right\rangle_{\mathbf{C}} \widetilde{\left(0, f_{\ell}\right)} f\right)^{2} \geq \rho_{2} \Gamma^{Z}(f) .
$$

Lemma 4.3. For any smooth cylinder function $f$,

$$
\begin{gathered}
\Gamma_{2}^{Z}(f)=\sum_{j=1}^{\infty} \sum_{\ell=1}^{N}\left(\widetilde{\left(e_{j}, 0\right)} \widetilde{\left(0, f_{\ell}\right)} f\right)^{2} \text { and } \\
\sum_{j=1}^{\infty}\left(\sum_{i=1}^{\infty} \widetilde{\left(e_{i}, 0\right)}\left(0, \widetilde{\omega\left(e_{i}, e_{j}\right)}\right) f\right)^{2} \leq\|\omega\|_{2}^{2} \Gamma_{2}^{Z}(f) .
\end{gathered}
$$

Similarly, for any $f \in C^{\infty}\left(G_{P}\right)$,

$$
\begin{aligned}
& \Gamma_{2, P}^{Z}(f)=\sum_{j=1}^{n} \sum_{\ell=1}^{N}\left(\widetilde{\left(e_{j}, 0\right)} \widetilde{\left(0, f_{\ell}\right)} f\right)^{2} \text { and } \\
& \left.\sum_{j=1}^{n}\left(\sum_{i=1}^{n} \widetilde{\left(e_{i}, 0\right)}\left(0, \widetilde{\omega\left(e_{i}, e_{j}\right.}\right)\right) f\right)^{2} \leq\|\omega\|_{2, P}^{2} \Gamma_{2, P}^{Z}(f) .
\end{aligned}
$$

Proof. We find $\Gamma_{2}^{Z}(f)$ by a straightforward calculation:

$$
\begin{aligned}
& \Gamma_{2}^{Z}(f)=\frac{1}{2} \sum_{j=1}^{\infty}{\widetilde{\left(e_{j}, 0\right)}}^{2} \sum_{\ell=1}^{N}\left(\widetilde{\left(0, f_{\ell}\right)} f\right)^{2}-\sum_{\ell=1}^{N} \sum_{j=1}^{\infty}\left(\widetilde{\left(0, f_{\ell}\right) f}\right)\left(\widetilde{\left(0, f_{\ell}\right)\left(e_{j}, 0\right)}{ }^{2} f\right) \\
& =\sum_{j=1}^{\infty} \sum_{\ell=1}^{N}\left\{\left(\widetilde{\left(e_{j}, 0\right)} \widetilde{\left(0, f_{\ell}\right)} f\right)^{2}+\left(\widetilde{\left(0, f_{\ell}\right)} f\right)\left({\widetilde{\left(e_{j}, 0\right)}}^{2} \widetilde{\left(0, f_{\ell}\right) f}\right)\right\} \\
& -\sum_{\ell=1}^{N} \sum_{j=1}^{\infty}\left(\widetilde{\left(0, f_{\ell}\right)} f\right)\left(\widetilde{\left(0, f_{\ell}\right)}{\widetilde{\left(e_{j}, 0\right)}}^{2} f\right) \\
& =\sum_{j=1}^{\infty} \sum_{\ell=1}^{N}\left(\widetilde{\left(e_{j}, 0\right)} \widetilde{\left(0, f_{\ell}\right)} f\right)^{2}
\end{aligned}
$$


where we have used that $\widetilde{\left(0, f_{\ell}\right)}$ and $\widetilde{\left(e_{j}, 0\right)}$ commute by equation $(\underline{3.8})$. The CauchySchwarz inequality then implies that

$$
\begin{aligned}
\sum_{j=1}^{\infty} & \left(\sum_{i=1}^{\infty} \widetilde{\left(e_{i}, 0\right)}\left(0, \widetilde{\omega\left(e_{i}, e_{j}\right)}\right) f\right)^{2} \\
& =\sum_{j=1}^{\infty}\left(\sum_{i=1}^{\infty} \sum_{\ell=1}^{N}\left\langle\omega\left(e_{i}, e_{j}\right), f_{\ell}\right\rangle_{\mathbf{C}} \widetilde{\left(e_{i}, 0\right)} \widetilde{\left(0, f_{\ell}\right) f}\right)^{2} \\
& \leq \sum_{j=1}^{\infty}\left(\sum_{i=1}^{\infty} \sum_{\ell=1}^{N}\left\langle\omega\left(e_{i}, e_{j}\right), f_{\ell}\right\rangle_{\mathbf{C}}^{2}\right)\left(\sum_{i=1}^{\infty} \sum_{\ell=1}^{N}\left(\widetilde{\left(e_{i}, 0\right)\left(\widetilde{\left.0, f_{\ell}\right)} f\right)^{2}}\right)=\|\omega\|_{2}^{2} \Gamma_{2}^{Z}(f) .\right.
\end{aligned}
$$

Lemmas 4.2 and 4.3 combine to give the desired curvature-dimension bound.

Proposition 4.4. For any $\nu>0$ and smooth cylinder function $f$,

$$
\Gamma_{2}(f)+\nu \Gamma_{2}^{Z}(f) \geq \frac{\rho_{2}}{4} \Gamma^{Z}(f)-\frac{\|\omega\|_{2}^{2}}{\nu} \Gamma(f) .
$$

Similarly, for any $\nu>0$ and $f \in C^{\infty}\left(G_{P}\right)$,

$$
\Gamma_{2, P}(f)+\nu \Gamma_{2, P}^{Z}(f) \geq \frac{\rho_{2, P}}{4} \Gamma_{P}^{Z}(f)-\frac{\|\omega\|_{2, P}^{2}}{\nu} \Gamma_{P}(f) .
$$

Proof. Again, we prove only the first inequality. To do this, we first use Lemma 4.2 and the commutation relation in (3.4) to estimate $\sum_{i, j=1}^{\infty}\left(\widetilde{\left(e_{i}, 0\right)} \widetilde{\left(e_{j}, 0\right)} f\right)^{2}$. In what follows, we also use the antisymmetry of the form $\omega$ :

$$
\begin{aligned}
& \sum_{i, j=1}^{\infty}\left(\widetilde{\left(e_{i}, 0\right)} \widetilde{\left(e_{j}, 0\right)} f\right)^{2}
\end{aligned}
$$

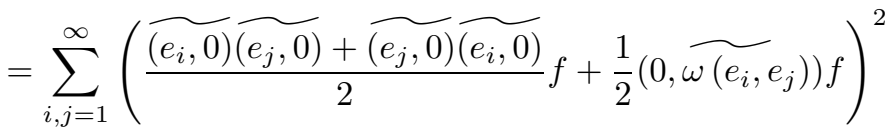

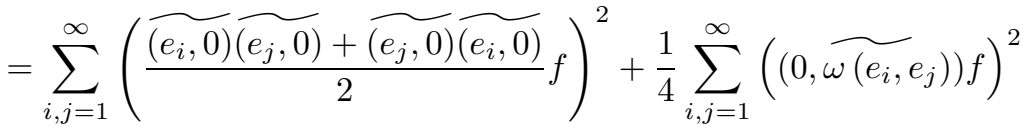

$$
\begin{aligned}
& +\frac{1}{2} \sum_{i, j=1}^{\infty}\left(\widetilde{\left(e_{i}, 0\right)} \widetilde{\left(e_{j}, 0\right)} f+\widetilde{\left(e_{j}, 0\right)} \widetilde{\left(e_{i}, 0\right)} f\right)\left(\left(0, \widetilde{\omega\left(e_{i}, e_{j}\right)}\right) f\right)
\end{aligned}
$$

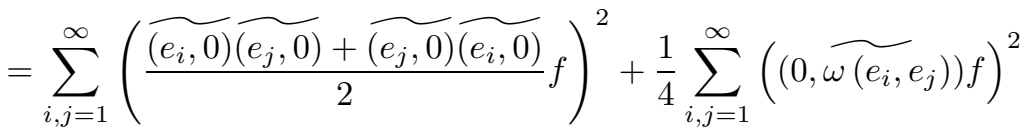

$$
\begin{aligned}
& \left.=\left\|\nabla_{H}^{2} f\right\|^{2}+\frac{1}{4} \sum_{i, j=1}^{\infty}\left(\left(0, \widetilde{\omega\left(e_{i}, e_{j}\right.}\right)\right) f\right)^{2},
\end{aligned}
$$

where

$$
\nabla_{H}^{2} f:=\sum_{i, j=1}^{\infty} \frac{\widetilde{\left(e_{i}, 0\right)} \widetilde{\left(e_{j}, 0\right) f}+\widetilde{\left(e_{j}, 0\right)}\left(\widetilde{\left(e_{i}, 0\right) f}\right.}{2}
$$


denotes the symmetrized Hessian. Thus by Lemma 4.2

$$
\left\|\nabla_{H}^{2} f\right\|^{2}+\frac{\rho_{2}}{4} \Gamma^{Z}(f) \leq \sum_{i, j=1}^{\infty}\left(\widetilde{\left(e_{i}, 0\right)} \widetilde{\left(e_{j}, 0\right)} f\right)^{2} \leq\left\|\nabla_{H}^{2} f\right\|^{2}+\frac{\|\omega\|_{2}^{2}}{4} \Gamma^{Z}(f) .
$$

Now we want to compute $\Gamma_{2}(f)$. The first term is simply

$$
\frac{1}{2} L \Gamma(f)=\sum_{i, j=1}^{\infty}\left({\widetilde{\left(e_{i}, 0\right)}}^{2} \widetilde{\left(e_{j}, 0\right) f}\right) \widetilde{\left(e_{j}, 0\right)} f+\left(\widetilde{\left(e_{i}, 0\right)} \widetilde{\left(e_{j}, 0\right) f}\right)^{2} .
$$

The second term may be expanded by applying (3.4) twice as follows:

$$
\begin{aligned}
& \Gamma(f, L f)=\sum_{i, j=1}^{\infty}\left(\widetilde{\left(e_{j}, 0\right)}{\widetilde{\left(e_{i}, 0\right)}}^{2} f\right) \widetilde{\left(e_{j}, 0\right) f} \\
& =\sum_{i, j=1}^{\infty}\left(\widetilde{\left(e_{i}, 0\right)} \widetilde{\left(e_{j}, 0\right) f}\right) \widetilde{\left(e_{j}, 0\right) f}-2 \widetilde{\left(\widetilde{\left(e_{i}, 0\right)}\left(0, \widetilde{\omega\left(e_{i}, e_{j}\right.}\right) f\right) \widetilde{\left(e_{j}, 0\right) f} .}
\end{aligned}
$$

Thus, by the lower bound in (4.3) we have that

$$
\begin{aligned}
\Gamma_{2}(f) & =\frac{1}{2}(L \Gamma(f)-2 \Gamma(f, L f)) \\
& =\sum_{i, j=1}^{\infty}\left(\widetilde{\left(e_{i}, 0\right)} \widetilde{\left(e_{j}, 0\right)} f\right)^{2}+2 \sum_{i, j=1}^{\infty} \widetilde{\left.\left(\widetilde{\left(e_{i}, 0\right)}\left(0, \widetilde{\omega\left(e_{i}, e_{j}\right.}\right)\right) f\right) \widetilde{\left(e_{j}, 0\right) f}} \\
& \left.\geq\left\|\nabla_{H}^{2} f\right\|^{2}+\frac{\rho_{2}}{4} \Gamma^{Z}(f)+2 \sum_{i, j=1}^{\infty}\left(\widetilde{\left(e_{i}, 0\right)}\left(0, \widetilde{\omega\left(e_{i}, e_{j}\right.}\right)\right) f\right) \widetilde{\left(e_{j}, 0\right) f}
\end{aligned}
$$

The Cauchy-Schwarz inequality now implies that for any $\nu>0$,

$$
\begin{aligned}
& \left.2 \mid \sum_{i, j=1}^{\infty} \widetilde{\left(\widetilde{\left.e_{i}, 0\right)}\right.}\left(0, \widetilde{\omega\left(e_{i}, e_{j}\right)}\right) f\right) \widetilde{\left(e_{j}, 0\right) f} \mid \\
& \left.\leq \nu \sum_{j=1}^{\infty}\left(\sum_{i=1}^{\infty} \widetilde{\left(e_{i}, 0\right)}\left(0, \widetilde{\omega\left(e_{i}, e_{j}\right.}\right)\right) f\right)^{2}+\frac{1}{\nu} \sum_{j=1}^{\infty}\left(\widetilde{\left(e_{j}, 0\right) f}\right)^{2} \\
& \leq \nu\|\omega\|_{2}^{2} \Gamma_{2}^{Z}(f)+\frac{1}{\nu} \Gamma(f),
\end{aligned}
$$

where the last inequality follows from Lemma 4.3. Combining (4.4) and (4.5) then gives

$$
\begin{aligned}
\Gamma_{2}(f)+\nu\|\omega\|_{2}^{2} \Gamma_{2}^{Z}(f) & \geq\left\|\nabla_{H}^{2} f\right\|^{2}+\frac{\rho_{2}}{4} \Gamma^{Z}(f)-\frac{1}{\nu} \Gamma(f) \\
& \geq \frac{\rho_{2}}{4} \Gamma^{Z}(f)-\frac{1}{\nu} \Gamma(f) .
\end{aligned}
$$

Finally, taking $\nu$ to be $\frac{\nu}{\|\omega\|_{2}^{2}}$ yields (4.2).

Remark 4.5. In the finite-dimensional case, one could use the Cauchy-Schwarz inequality in (4.6) to give a lower bound on $\left\|\nabla_{H}^{2} f\right\|^{2}$ by $(L f)^{2}$ with a coefficient depending on $\operatorname{dim}(H)$. Such an estimate standardly leads, for example, to Li-Yau type Harnack inequalities and bounds on logarithmic derivatives of the heat kernel. See for example [2, 8, 27]. 
We now prove that the desired commutation formula holds trivially on $G$ and $G_{P}$.

Lemma 4.6. For any smooth cylinder function $f$ on $G$,

$$
\Gamma\left(f, \Gamma^{Z}(f)\right)=\Gamma^{Z}(f, \Gamma(f)) .
$$

Similarly, for any $f \in C^{\infty}\left(G_{P}\right)$,

$$
\Gamma_{P}\left(f, \Gamma_{P}^{Z}(f)\right)=\Gamma_{P}^{Z}\left(f, \Gamma_{P}(f)\right) .
$$

Proof. The proof is a straightforward computation:

$$
\begin{aligned}
\Gamma\left(f, \Gamma^{Z}(f)\right) & =\sum_{j=1}^{\infty}\left(\widetilde{\left(e_{j}, 0\right) f}\right)\left(\widetilde{\left(e_{j}, 0\right)} \Gamma^{Z}(f)\right) \\
& =\sum_{j=1}^{\infty} \sum_{\ell=1}^{N}\left(\widetilde{\left(e_{j}, 0\right)} f\right)\left(\widetilde{\left(e_{j}, 0\right)}\left(\widetilde{\left(0, f_{\ell}\right)} f\right)^{2}\right) \\
& =2 \sum_{j=1}^{\infty} \sum_{\ell=1}^{N}\left(\widetilde{\left(e_{j}, 0\right)} f\right)\left(\widetilde{\left(0, f_{\ell}\right)} f\right)\left(\widetilde{\left(e_{j}, 0\right)}\left(\widetilde{\left.0, f_{\ell}\right)} f\right)\right. \\
& =2 \sum_{j=1}^{\infty} \sum_{\ell=1}^{N}\left(\widetilde{\left(0, f_{\ell}\right)} f\right)\left(\widetilde{\left(e_{j}, 0\right)} f\right)\left(\widetilde{\left(f_{\ell}, 0\right)}\left(\widetilde{\left(0, e_{j}\right)} f\right)\right) \\
& =\Gamma^{Z}(f, \Gamma(f)),
\end{aligned}
$$

where we have again used the commutativity of $\widetilde{\left(e_{j}, 0\right)}$ and $\widetilde{\left(0, f_{l}\right)}$ in the penultimate equality. The computation for the second equality is completely analogous.

4.2. Functional inequalities on $G_{P}$. Again note that for any $P \in \operatorname{Proj}(W)$, $G_{P}=P H \times \mathbf{C}$ is a finite-dimensional step 2 stratified Lie group. If $\left\{e_{j}\right\}_{j=1}^{n}$ is an orthonormal basis of $P H$, then $\left\{\widetilde{\left(e_{j}, 0\right)}\right\}_{j=1}^{n}$ is a Hörmander set of vector fields on $G_{P}$. Thus we may apply the results of Section 2 to $G_{P}$. In particular, by Proposition 2.5, the curvature bound for $G_{P}$ found in Proposition 4.4 implies the following reverse Poincaré inequality holds on all $G_{P}$. Here, we let $L_{P}=\sum_{j=1}^{n}{\widetilde{\left(e_{j}, 0\right)}}^{2}$ and $\left\{P_{t}^{P}\right\}_{t>0}$ denote the associated semi-group. Also, define the function classes $\mathcal{C}_{P}$ and $\mathcal{C}_{P}^{+}$analogously for $G_{P}$ as was done in Notation 2.3.

Proposition 4.7 (Reverse Poincaré inequality). For any $P \in \operatorname{Proj}(W), T>0$, and $f \in \mathcal{C}_{P}$,

$$
\Gamma_{P}\left(P_{T}^{P} f\right)+\rho_{2, P} T \Gamma_{P}^{Z}\left(P_{T}^{P} f\right) \leq \frac{1+\frac{8\|\omega\|_{2, P}^{2}}{\rho_{2, P}}}{2 T}\left(P_{T}^{P}\left(f^{2}\right)-\left(P_{T}^{P} f\right)^{2}\right) .
$$

In particular, the following reverse Poincaré inequality holds:

$$
\Gamma_{P}\left(P_{T}^{P} f\right) \leq \frac{1+\frac{8\|\omega\|_{2, P}^{2}}{\rho_{2, P}}}{2 T}\left(P_{T}^{P}\left(f^{2}\right)-\left(P_{T}^{P} f\right)^{2}\right) .
$$

Similarly, Theorem 2.7 implies that Proposition 4.4, coupled with the commutation relation of Lemma 4.6, give the following reverse log Sobolev inequality on all $G_{P}$. 
Theorem 4.8 (Reverse $\log$ Sobolev inequality). For any $P \in \operatorname{Proj}(W), T>0$, and $f \in \mathcal{C}_{P}^{+}$,

$$
\Gamma_{P}\left(\ln P_{T}^{P} f\right)+\rho_{2, P} T \Gamma_{P}^{Z}\left(\ln P_{T}^{P} f\right) \leq \frac{1+\frac{8\|\omega\|_{2, P}^{2}}{\rho_{2, P}}}{T}\left(\frac{P_{T}^{P}(f \ln f)}{P_{T}^{P} f}-\ln P_{T}^{P} f\right) .
$$

In particular, the following reverse log Sobolev inequality holds:

$$
\Gamma_{P}\left(\ln P_{T}^{P} f\right) \leq \frac{1+\frac{8\|\omega\|_{2, P}^{2}}{\rho_{2, P}}}{T}\left(\frac{P_{T}^{P}(f \ln f)}{P_{T}^{P} f}-\ln P_{T}^{P} f\right) .
$$

The combination of Proposition 2.10 with the reverse log Sobolev inequality found in Theorem 4.8 implies that the following Harnack type inequalities hold on each $G_{P}$.

Proposition 4.9 (Wang type Harnack inequality). Let $P \in \operatorname{Proj}(W)$. Then, for all $T>0, x, y \in G_{P}, f \in L^{\infty}\left(G_{P}\right)$ with $f \geq 0$, and $p \in(1, \infty)$,

$$
\left(P_{T}^{P} f\right)^{p}(x) \leq P_{T}^{P} f^{p}(y) \exp \left(\left(1+\frac{8\|\omega\|_{2, P}^{2}}{\rho_{2, P}}\right) \frac{p d_{P}^{2}(x, y)}{4(p-1) T}\right) .
$$

\section{Heat Kernel measure on $G$ AND a QUASI-INVARIANCE THEOREM}

In this section, we show how the Wang type Harnack inequalities on $G_{P}$ obtained in the previous section lead to the quasi-invariance of the subelliptic heat kernel measure on $G$. First, we must of course define the heat kernel measure on $G$, which we define as the end point distribution of a Brownian motion.

5.1. Brownian motion on $G$. We define a "subelliptic" Brownian motion $\left\{g_{t}\right\}_{t \geq 0}$ on $G$ and collect its various properties. The primary references for this section are Sections 4 of [15] and [17] and Section 2.5 of [19. Any statements made here without proof are proved in these references.

Let $\left\{B_{t}\right\}_{t \geq 0}$ be a Brownian motion on $W$ with variance determined by

$$
\mathbb{E}\left[\left\langle B_{s}, h\right\rangle_{H}\left\langle B_{t}, k\right\rangle_{H}\right]=\langle h, k\rangle_{H} \min (s, t),
$$

for all $s, t \geq 0$ and $h, k \in H_{*}$. The following is Proposition 4.1 of [15], and this result implicitly relies on the fact that Lemma 3.7 implies that the bilinear form $\omega$ is a Hilbert-Schmidt.

Proposition 5.1. For $P \in \operatorname{Proj}(W)$, let $M_{t}^{P}$ denote the continuous $L^{2}$-martingale on $\mathbf{C}$ defined by

$$
M_{t}^{P}=\int_{0}^{t} \omega\left(P B_{s}, d P B_{s}\right) .
$$

In particular, if $\left\{P_{n}\right\}_{n=1}^{\infty} \subset \operatorname{Proj}(W)$ is an increasing sequence of projections and $M_{t}^{n}:=M_{t}^{P_{n}}$, then there exists an $L^{2}$-martingale $\left\{M_{t}\right\}_{t \geq 0}$ in $\mathbf{C}$ such that, for all $p \in[1, \infty)$ and $t>0$,

$$
\lim _{n \rightarrow \infty} \mathbb{E}\left[\sup _{\tau \leq t}\left\|M_{\tau}^{n}-M_{\tau}\right\|_{\mathbf{C}}^{p}\right]=0,
$$

and $M_{t}$ is independent of the sequence of projections. 
As $M_{t}$ is independent of the defining sequence of projections, we will denote the limiting process by

$$
M_{t}=\int_{0}^{t} \omega\left(B_{s}, d B_{s}\right) .
$$

Definition 5.2. The continuous $G$-valued process given by

$$
g_{t}=\left(B_{t}, \frac{1}{2} M_{t}\right)=\left(B_{t}, \frac{1}{2} \int_{0}^{t} \omega\left(B_{s}, d B_{s}\right)\right)
$$

is a Brownian motion on $G$. For $t>0$, let $\nu_{t}=\operatorname{Law}\left(g_{2 t}\right)$ denote the heat kernel measure at time $2 t$ on $G$.

We include the following proposition (see [19, Proposition 2.30]) which states that, as the name suggests, the Cameron-Martin subgroup is a subspace of heat kernel measure 0 .

Proposition 5.3. For all $t>0, \nu_{t}\left(G_{C M}\right)=0$.

Proposition 5.1, along with the fact that, for all $p \in[1, \infty)$ and $t>0$,

$$
\lim _{n \rightarrow \infty} \mathbb{E}\left[\sup _{\tau \leq t}\left\|B_{\tau}-P_{n} B_{\tau}\right\|_{W}^{p}\right]=0
$$

(see for example Proposition 4.6 of [15]), makes the following proposition clear.

Proposition 5.4. For $P \in \operatorname{Proj}(W)$, let $g_{t}^{P}$ be the continuous process on $G_{P}$ defined by

$$
g_{t}^{P}=\left(P B_{t}, \frac{1}{2} \int_{0}^{t} \omega\left(P B_{s}, d P B_{s}\right)\right) .
$$

Then $g_{t}^{P}$ is a Brownian motion on $G_{P}$. In particular, let $\left\{P_{n}\right\}_{n=1}^{\infty} \subset \operatorname{Proj}(W)$ be increasing projections and $g_{t}^{n}:=g_{t}^{P_{n}}$. Then, for all $p \in[1, \infty)$ and $t>0$,

$$
\lim _{n \rightarrow \infty} \mathbb{E}\left[\sup _{\tau \leq t}\left\|g_{\tau}^{n}-g_{\tau}\right\|_{\mathfrak{g}}^{p}\right]=0 .
$$

Notation 5.5. For all $P \in \operatorname{Proj}(W)$ and $t>0$, let $\nu_{t}^{P}:=\operatorname{Law}\left(g_{2 t}^{P}\right)$, and for all $n \in \mathbb{N}$ let $\nu_{t}^{n}:=\operatorname{Law}\left(g_{2 t}^{n}\right)=\operatorname{Law}\left(g_{2 t}^{P_{n}}\right)$.

Proposition 5.6. Let $L$ be as defined in Proposition 3.19. Then we will call $L$ the subelliptic Laplacian, and $\frac{1}{2} L$ is the generator for $\left\{g_{t}\right\}_{t \geq 0}$ so that, for any smooth cylinder function $f: G \rightarrow \mathbb{R}$,

$$
f\left(g_{t}\right)-\frac{1}{2} \int_{0}^{t} L f\left(g_{s}\right) d s
$$

is a local martingale.

Corollary 5.7. Let $f=F \circ \pi_{P}$ be a cylinder function on $G$ such that $F \in C^{2}\left(G_{P}\right)$, and there exist $K>0$ and $p<\infty$ such that

$$
|F(h, c)|+\left\|F^{\prime}(h, c)\right\|+\left\|F^{\prime \prime}(h, c)\right\| \leq K\left(1+\|h\|_{P H}+\|c\|_{\mathbf{C}}\right)^{p},
$$

for all $(h, c) \in G_{P}$. Then

$$
\mathbb{E}\left[f\left(g_{t}\right)\right]=f(e)+\frac{1}{2} \int_{0}^{t} \mathbb{E}\left[(L f)\left(g_{s}\right)\right] d s .
$$


That is,

is a weak solution to the heat equation

$$
\nu_{t}(f):=\int_{0}^{t} f d \nu_{s}=f(e)+\int_{0}^{t} \nu_{s}(L f) d s
$$

$$
\partial_{t} \nu_{t}=L \nu_{t}, \quad \text { with } \lim _{t \downarrow 0} \nu_{t}=\delta_{e} .
$$

For all projections satisfying Hörmander's condition, the Brownian motions on $G_{P}$ are subelliptic diffusions and thus their laws are absolutely continuous with respect to the finite-dimensional reference measure and their transition kernels are smooth. The following is Lemma 2.27 of 19 .

Lemma 5.8. For all $P \in \operatorname{Proj}(W)$ and $t>0$, we have $\nu_{t}^{P}(d x)=p_{t}^{P}(x) d x$, where $d x$ is the Riemannian volume measure (equal to Haar measure) and $p_{t}^{P}(x)$ is the heat kernel on $G_{P}$.

5.2. Quasi-invariance and Radon-Nikodym derivative estimates. For now, let us fix $P \in \operatorname{Proj}(W)$, and recall that by Proposition 4.9, for all $T>0, x, y \in G_{P}$, $f \geq 0$, and $p \in(1, \infty)$,

$$
\left(P_{T}^{P} f\right)^{p}(x) \leq P_{T}^{P} f^{p}(y) \exp \left(\left(1+\frac{8\|\omega\|_{2, P}^{2}}{\rho_{2, P}}\right) \frac{p d_{P}^{2}(x, y)}{4(p-1) T}\right) .
$$

Then Lemma 2.11 implies that this estimate is equivalent to

$$
\left(\int_{G}\left[\frac{p_{T}^{P}(y, z)}{p_{T}^{P}(x, z)}\right]^{1 /(p-1)} p_{T}^{P}(y, z) d z\right)^{p-1} \leq \exp \left(\left(1+\frac{8\|\omega\|_{2, P}^{2}}{\rho_{2, P}}\right) \frac{p d_{P}^{2}(x, y)}{4(p-1) T}\right),
$$

where $p_{T}^{P}$ is the heat kernel on $G_{P}$. In particular, for $q=p /(p-1) \in(1, \infty)$, this implies that

$$
\left(\int_{G}\left[\frac{p_{T}^{P}(y, z)}{p_{T}^{P}(x, z)}\right]^{q} p_{T}^{P}(x, z) d z\right)^{1 / q} \leq \exp \left(\left(1+\frac{8\|\omega\|_{2, P}^{2}}{\rho_{2, P}}\right) \frac{(q-1) d_{P}^{2}(x, y)}{4 T}\right) .
$$

Using the properties of heat kernels on finite-dimensional groups given in Lemma 2.1, we have that

$$
\begin{aligned}
\int_{G}\left[\frac{p_{T}^{P}(y, z)}{p_{T}^{P}(x, z)}\right]^{q} p_{T}^{P}(x, z) d z & =\int_{G}\left[\frac{p_{T}^{P}\left(y z^{-1}\right)}{p_{T}^{P}\left(x z^{-1}\right)}\right]^{q} p_{T}^{P}\left(x z^{-1}\right) d z \\
& =\int_{G}\left[\frac{p_{T}^{P}\left(z y^{-1}\right)}{p_{T}^{P}\left(z x^{-1}\right)}\right]^{q} p_{T}^{P}\left(z x^{-1}\right) d z .
\end{aligned}
$$

Then, for $x=e$, we may rewrite inequality (5.1) as

$$
\left(\int_{G}\left[\frac{p_{T}^{P}\left(z y^{-1}\right)}{p_{T}^{P}(z)}\right]^{q} p_{T}^{P}(z) d z\right)^{1 / q} \leq \exp \left(\left(1+\frac{8\|\omega\|_{2, P}^{2}}{\rho_{2, P}}\right) \frac{(q-1) d_{P}^{2}(e, y)}{4 T}\right) .
$$

Now, for $y \in G_{P}$, again we let $R_{y}: G_{P} \rightarrow G_{P}$ denote right translation. Then $\nu_{T}^{P} \circ R_{y}^{-1}$ is the push forward of $\nu_{T}$ under $R_{y}$. For fixed $T>0$, let $J_{P}^{r}$ denote the Radon-Nikodym derivative of $\nu_{T}^{P} \circ R_{y}^{-1}$ with respect to $\nu_{T}^{P}$. Then (5.2) is equivalent to

$$
\left\|J_{P}^{r}\right\|_{L^{q}\left(G_{P}, \nu_{T}^{P}\right)} \leq \exp \left(\left(1+\frac{8\|\omega\|_{2, P}^{2}}{\rho_{2, P}}\right) \frac{(q-1) d_{P}^{2}(e, y)}{4 T}\right) .
$$


Alternatively, again using the properties of $p_{T}^{P}$ described in Lemma 2.1 and the translation invariance of Haar measure, we could write

$$
\begin{aligned}
\int_{G}\left[\frac{p_{T}^{P}(y, z)}{p_{T}^{P}(x, z)}\right]^{q} p_{T}^{P}(x, z) d z & =\int_{G}\left[\frac{p_{T}^{P}\left(y^{-1} z\right)}{p_{T}^{P}\left(x^{-1} z\right)}\right]^{q} p_{T}^{P}\left(x^{-1} z\right) d z \\
& =\int_{G}\left[\frac{p_{T}^{P}\left(y^{-1} x z\right)}{p_{T}^{P}(z)}\right]^{q} p_{T}^{P}(z) d z
\end{aligned}
$$

Then taking $x=e$ and combining this with the inequality (5.1) gives

$$
\left(\int_{G}\left[\frac{p_{T}^{P}\left(y^{-1} z\right)}{p_{T}^{P}(z)}\right]^{q} p_{T}^{P}(z) d z\right)^{1 / q} \leq \exp \left(\left(1+\frac{8\|\omega\|_{2, P}^{2}}{\rho_{2, P}}\right) \frac{(q-1) d_{P}^{2}(e, y)}{4 T}\right),
$$

which is equivalent to the left translation analogue

$$
\left\|J_{P}^{l}\right\|_{L^{q}\left(G_{P}, \nu_{T}^{P}\right)} \leq \exp \left(\left(1+\frac{8\|\omega\|_{2, P}^{2}}{\rho_{2, P}}\right) \frac{(q-1) d_{P}^{2}(e, y)}{4 T}\right),
$$

where $L_{y}: G_{P} \rightarrow G_{P}$ is left translation, $\nu_{T}^{P} \circ L_{y}^{-1}$ is the push forward of $\nu_{T}^{P}$ under $L_{y}$, and $J_{P}^{l}$ denotes the Radon-Nikodym derivative of $\nu_{T}^{P} \circ L_{y}^{-1}$ with respect to $\nu_{T}$.

Such estimates on the finite-dimensional projection groups $G_{P}$ may be used to prove a quasi-invariance theorem on the infinite-dimensional group $G$. The following proof is analogous to the proofs of Theorem 7.2 and 7.3 in [16. Similar methods were also used for the elliptic setting in loop groups in [13], in infinite-dimensional Heisenberg-like groups in [15], and in semi-infinite Lie groups in [30].

Theorem 5.9 (Quasi-invariance of $\nu_{t}$ ). For all $y \in G_{C M}$ and $T>0, \nu_{T}$ is quasiinvariant under left and right translations by $y$. Moreover, for all $q \in(1, \infty)$,

$$
\left\|\frac{d\left(\nu_{T} \circ R_{y}^{-1}\right)}{d \nu_{T}}\right\|_{L^{q}\left(G, \nu_{T}\right)} \leq \exp \left(\left(1+\frac{8\|\omega\|_{2}^{2}}{\rho_{2}}\right) \frac{(q-1) d^{2}(e, y)}{4 T}\right)
$$

and

$$
\left\|\frac{d\left(\nu_{T} \circ L_{y}^{-1}\right)}{d \nu_{T}}\right\|_{L^{q}\left(G, \nu_{T}\right)} \leq \exp \left(\left(1+\frac{8\|\omega\|_{2}^{2}}{\rho_{2}}\right) \frac{(q-1) d^{2}(e, y)}{4 T}\right) .
$$

Proof. Fix $T>0$ and $P_{0} \in \operatorname{Proj}(W)$. Let $y \in G_{0}$ and $\left\{P_{n}\right\}_{n=1}^{\infty}$ be an increasing sequence of projections such that $P_{0} H \subset P_{n} H$ for all $n$ and $\left.P_{n}\right|_{H} \uparrow I_{H}$. Let $J_{n}^{r}:=J_{P_{n}}^{r}$ denote the Radon-Nikodym derivative of $\nu_{T}^{n} \circ R_{y}^{-1}$ with respect to $\nu_{T}^{n}$. Then by the previous discussion and (5.3), we have

$$
\left\|J_{n}^{r}\right\|_{L^{q}\left(G_{n}, \nu_{T}^{n}\right)} \leq \exp \left(\left(1+\frac{8\|\omega\|_{2, n}^{2}}{\rho_{2, n}}\right) \frac{(q-1) d_{n}^{2}(e, y)}{4 T}\right),
$$

where we let $\|\omega\|_{2, n}:=\|\omega\|_{2, P_{n}}$ and $\rho_{2, n}=\rho_{2, P_{n}}$. 
By Proposition 5.4, we have that for any bounded continuous $f$ on $G$,

$$
\int_{G} f d \nu_{T}=\lim _{n \rightarrow \infty} \int_{G_{n}} f \circ i_{n} d \nu_{T}^{n}
$$

where $i_{n}: G_{n} \rightarrow G$ denotes the inclusion map. Note that

$$
\begin{aligned}
& \int_{G_{n}}\left|\left(f \circ i_{n}\right)(x y)\right| d \nu_{T}^{n}(x)=\int_{G_{n}} J_{n}^{r}(x)\left|\left(f \circ i_{n}\right)(x)\right| d \nu_{T}^{n}(x) \\
& \leq\left\|f \circ i_{n}\right\|_{L^{q^{\prime}}\left(G_{n}, \nu_{T}^{n}\right)} \exp \left(\left(1+\frac{8\|\omega\|_{2, n}^{2}}{\rho_{2, n}}\right) \frac{(q-1) d_{n}^{2}(e, y)}{4 T}\right),
\end{aligned}
$$

where $q^{\prime}$ is the conjugate exponent to $q$. Allowing $n \rightarrow \infty$ in this last inequality yields

$$
\int_{G}|f(x y)| d \nu_{T}(x) \leq\|f\|_{L^{q^{\prime}}\left(G, \nu_{T}\right)} \exp \left(\left(1+\frac{8\|\omega\|_{2}^{2}}{\rho_{2}}\right) \frac{(q-1) d^{2}(e, y)}{4 T}\right),
$$

by Lemma 3.25, Remark 4.1, and equation (5.6). Thus, we have proved that (5.7) holds for $f \in B C(G)$ and $y \in \bigcup_{P \in \operatorname{Proj}(W)} G_{P}$. As this union is dense in $G_{C M}$ by Proposition 3.23, dominated convergence along with the continuity of $d(e, y)$ in $y$ implies that (5.7) holds for all $y \in G_{C M}$.

Since the bounded continuous functions are dense in $L^{q^{\prime}}\left(G, \nu_{T}\right)$ (see for example Theorem A.1 of [24), the inequality in (5.7) implies that the linear functional $\varphi_{y}: B C(G) \rightarrow \mathbb{R}$ defined by

$$
\varphi_{y}(f)=\int_{G} f(x y) d \nu_{T}(x)
$$

has a unique extension to an element, still denoted by $\varphi_{y}$, of $L^{q^{\prime}}\left(G, \nu_{T}\right)^{*}$ which satisfies the bound

$$
\left|\varphi_{y}(f)\right| \leq\|f\|_{L^{q^{\prime}}\left(G, \nu_{T}\right)} \exp \left(\left(1+\frac{8\|\omega\|_{2}^{2}}{\rho_{2}}\right) \frac{(q-1) d^{2}(e, y)}{4 T}\right)
$$

for all $f \in L^{q^{\prime}}\left(G, \nu_{T}\right)$. Since $L^{q^{\prime}}\left(G, \nu_{T}\right)^{*} \cong L^{q}\left(G, \nu_{T}\right)$, there then exists a function $J_{y}^{r} \in L^{q}\left(G, \nu_{T}\right)$ such that

$$
\varphi_{y}(f)=\int_{G} f(x) J_{y}^{r}(x) d \nu_{T}(x)
$$

for all $f \in L^{q^{\prime}}\left(G, \nu_{T}\right)$, and

$$
\left\|J_{y}^{r}\right\|_{L^{q}\left(G, \nu_{T}\right)} \leq \exp \left(\left(1+\frac{8\|\omega\|_{2}^{2}}{\rho_{2}}\right) \frac{(q-1) d^{2}(e, y)}{4 T}\right) .
$$

Now restricting (5.8) to $f \in B C(G)$, we may rewrite this equation as

$$
\int_{G} f(x) d \nu_{T}\left(x y^{-1}\right)=\int_{G} f(x) J_{y}^{r}(x) d \nu_{T}(x) .
$$

Then a monotone class argument (again using Theorem A.1 of [24]) shows that (5.9) is valid for all bounded measurable functions $f$ on $G$. Thus, $d\left(\nu_{T} \circ R_{y}^{-1}\right) / d \nu_{T}$ exists and is given by $J_{y}^{r}$, which is in $L^{q}$ for all $q \in(1, \infty)$ and satisfies the bound (5.5). 
A parallel argument employing the estimate in (5.4) gives the analogous result for $d\left(\nu_{T} \circ L_{y}^{-1}\right) / d \nu_{T}$. Alternatively, one could use the right translation invariance just proved along with the fact that $\nu_{T}$ inherits invariance under the inversion map $y \mapsto y^{-1}$ from its finite-dimensional projections and that $d\left(e, y^{-1}\right)=d(e, y)$.

We may now observe that, by the proof of Lemma 2.11, we have the following Wang type Harnack inequality on $G$. For $T>0, x \in G$, and $f \in L^{\infty}\left(G, \nu_{T}\right)$, let

$$
P_{T} f(x):=\mathbb{E}\left[f\left(x g_{2 T}\right)\right]=\int_{G} f(x y) d \nu_{T}(y),
$$

where $\left\{x g_{t}\right\}_{t \geq 0}$ is a Brownian motion on $G$ started at $x$.

Corollary 5.10 (Wang type Harnack inequality on $G$ ). Then for all $T>0$ and $x, y \in G_{C M}, f \in L^{\infty}\left(G, \nu_{T}\right)$ with $f \geq 0$, and $p \in(1, \infty)$,

$$
\left(P_{T} f\right)^{p}(x) \leq P_{T} f^{p}(y) \exp \left(\left(1+\frac{8\|\omega\|_{2}^{2}}{\rho_{2}}\right) \frac{p d^{2}(x, y)}{4(p-1) T}\right) .
$$

Our final result is the following corollary which concerns two points. First, we are interested in the smoothing properties of the semi-group $P_{T}$ in the absence of a reference measure. One way to approach this is to use a method similar to the proof of [35, Theorem 1.1]. A significant difference is that in [35] and some related work, the proof that the semi-group is strong Feller uses not only Harnack type inequalities, but also a version of Girsanov's theorem for the solution of the stochastic differential equation they consider. Note that we do not presently have path space quasi-invariance available in our degenerate case. The second point concerns the fact that the semi-group has smoothing properties, but only on the Cameron-Martin subgroup which has the heat kernel measure 0 as has been shown in [19, Proposition 2.30]. This is an infinite-dimensional phenomenon. In the flat abstract Wiener space setting such a phenomenon has been observed in [1]. In the context of holomorphic functions on complex abstract Wiener space, it has been proved in [32, 33] and on complex infinite-dimensional groups in [11, 15, 18]. The importance of the strong Feller property for probabilistic potential theory in infinite dimensions has been discussed in 21. Also, in 22 those authors explore the implications of a weaker version of the strong Feller property in a hypoelliptic setting.

Corollary 5.11 (Feller skeleton). For all $T>0$ and bounded continuous functions $f$ on $G$ such that $f \geq 0,\left(P_{T} f\right)(y) \rightarrow\left(P_{T} f\right)(x)$ as $d(x, y) \rightarrow 0$ for $x, y \in G_{C M}$.

Proof. Fix $T>0$ and $P_{0} \in \operatorname{Proj}(W)$. Let $y \in G_{0}$ and $\left\{P_{n}\right\}_{n=1}^{\infty}$ be an increasing sequence of projections such that $P_{0} H \subset P_{n} H$ for all $n$ and $\left.P_{n}\right|_{H} \uparrow I_{H}$. Then, for $P_{T}^{n}:=P_{T}^{P_{n}}$ and $p_{T}^{n}:=p_{T}^{P_{n}}$,

$$
\begin{aligned}
\left|\left(P_{T}^{n} f\right)(y)-\left(P_{T}^{n} f\right)(x)\right| & \leq \int_{G_{n}} f(z)\left|p_{T}^{n}(x, z)-p_{T}^{n}(y, z)\right| d z \\
& \leq\|f\|_{\infty} \int_{G_{n}}\left|p_{T}^{n}(x, z)-p_{T}^{n}(y, z)\right| d z
\end{aligned}
$$


We also have that

$$
\begin{aligned}
& \left(\int_{G_{n}}\left|p_{T}^{n}(x, z)-p_{T}^{n}(y, z)\right| d z\right)^{2}=\left(\int_{G_{n}}\left|\frac{p_{T}^{n}(x, z)}{p_{T}^{n}(y, z)}-1\right| p_{T}^{n}(y, z) d z\right)^{2} \\
& \quad \leq \int_{G_{n}}\left(\frac{p_{T}^{n}(x, z)}{p_{T}^{n}(y, z)}-1\right)^{2} p_{T}^{n}(y, z) d z=\int_{G_{n}}\left(\frac{p_{T}^{n}(x, z)}{p_{T}^{n}(y, z)}\right)^{2} p_{T}^{n}(y, z) d z-1 \\
& \quad \leq \exp \left(\left(1+\frac{8\|\omega\|_{2, n}^{2}}{\rho_{2, n}}\right) \frac{d_{n}^{2}(x, y)}{2 T}\right)-1
\end{aligned}
$$

by (5.1) with $q=2$. By (5.6) we may show that for any $x, y \in G_{C M}$,

$$
\begin{aligned}
& \left|\left(P_{T} f\right)(x)-\left(P_{T} f\right)(y)\right|^{2} \\
& \quad \leq\|f\|_{\infty}^{2}\left(\exp \left(\left(1+\frac{8\|\omega\|_{2}^{2}}{\rho_{2}}\right) \frac{d^{2}(x, y)}{2 T}\right)-1\right) \\
& \quad \leq\|f\|_{\infty}^{2}\left(1+\frac{8\|\omega\|_{2}^{2}}{\rho_{2}}\right) \frac{d^{2}(x, y)}{2 T} \exp \left(\left(1+\frac{8\|\omega\|_{2}^{2}}{\rho_{2}}\right) \frac{d^{2}(x, y)}{2 T}\right),
\end{aligned}
$$

since $e^{x}-1 \leq x e^{x}$ for any $x>0$. It is clear that the right-hand side tends to 0 when $d(x, y) \rightarrow 0$.

\section{ACKNOWLEDGEMENTS}

The authors are grateful to the anonymous referee for the careful reading of the manuscript and the useful remarks. The second author thanks M. Röckner, who asked about the Feller property in this context and thus inspired the inclusion of Corollary 5.11 .

\section{REFERENCES}

1. Shigeki Aida and Hiroshi Kawabi, Short time asymptotics of a certain infinite dimensional diffusion process, Stochastic analysis and related topics, VII (Kusadasi, 1998), Progr. Probab., vol. 48, Birkhäuser Boston, Boston, MA, 2001, pp. 77-124. MR1915450 (2003m:60219)

2. Dominique Bakry, Fabrice Baudoin, Michel Bonnefont, and Bin Qian, Subelliptic Li-Yau estimates on three dimensional model spaces, Potential theory and stochastics in Albac, Theta Ser. Adv. Math., vol. 11, Theta, Bucharest, 2009, pp. 1-10. MR.2681833 (2012b:58038)

3. Dominique Bakry and Michel Émery, Diffusions hypercontractives, Séminaire de probabilités, XIX, 1983/84, Lecture Notes in Math., vol. 1123, Springer, Berlin, 1985, pp. 177-206. MR889476 (88j:60131)

4. Dominique Bakry and Michel Ledoux, A logarithmic Sobolev form of the Li-Yau parabolic inequality, Rev. Mat. Iberoam. 22 (2006), no. 2, 683-702. MR2294794 (2008m:58051)

5. Dominique Bakry and Zhongmin M. Qian, Harnack inequalities on a manifold with positive or negative Ricci curvature, Rev. Mat. Iberoamericana 15 (1999), no. 1, 143-179. MR.1681640 (2000f:58052)

6. Fabrice Baudoin and Michel Bonnefont, Log-Sobolev inequalities for subelliptic operators satisfying a generalized curvature dimension inequality, Journ. of Funct. Anal. 262 (2012), no. 6, 2646-2676. MR2885961

7. Fabrice Baudoin, Michel Bonnefont and Nicola Garofalo, A sub-Riemannian curvaturedimension inequality, volume doubling property and the Poincaré inequality, arXiv:1007.1600, 2010 .

8. Fabrice Baudoin and Nicola Garofalo, Curvature-dimension inequalities and Ricci lower bounds for sub-Riemannian manifolds with transverse symmetries, arXiv:1101.3590v1, 2011.

9. Fabrice Baudoin and Josef Teichmann, Hypoellipticity in infinite dimensions and an application in interest rate theory, Ann. Appl. Probab. 15 (2005), no. 3, 1765-1777. MR2152244 (2006g:60080) 
10. Vladimir I. Bogachev, Gaussian measures, Mathematical Surveys and Monographs, vol. 62, American Mathematical Society, Providence, RI, 1998. MR,1642391 (2000a:60004)

11. Matthew Cecil, The Taylor map on complex path groups, J. Funct. Anal. 254 (2008), 318-367. MR2376574 (2009j:58056)

12. Bruce K. Driver, Towards calculus and geometry on path spaces, Stochastic analysis (Ithaca, NY, 1993), Proc. Sympos. Pure Math., vol. 57, Amer. Math. Soc., Providence, RI, 1995, pp. 405-422. MR.1335485 (96e:60097)

13. , Integration by parts and quasi-invariance for heat kernel measures on loop groups, J. Funct. Anal. 149 (1997), no. 2, 470-547. MR1472366 (99a:60054a)

14. - Heat kernels measures and infinite dimensional analysis, Heat kernels and analysis on manifolds, graphs, and metric spaces (Paris, 2002), Contemp. Math., vol. 338, Amer. Math. Soc., Providence, RI, 2003, pp. 101-141. MR2039953 (2005e:58058)

15. Bruce K. Driver and Maria Gordina, Heat kernel analysis on infinite-dimensional Heisenberg groups, Journal of Functional Analysis 255 (2008), no. 2, 2395-2461. MR2473262 (2010f:60010)

16. Integrated Harnack inequalities on Lie groups, J. Differential Geom. 83 (2009), no. 3, 501-550. MR2581356 (2011d:58086)

17. _ Square integrable holomorphic functions on infinite-dimensional Heisenberg type groups, Probability Theory and Related Fields 147 (2010), no. 3-4, 481-528. MR.2639713 (2011k:58052)

18. Maria Gordina, Heat kernel analysis and Cameron-Martin subgroup for infinite dimensional groups, J. Funct. Anal. 171 (2000), no. 1, 192-232. MR1742865 (2001g:60132)

19. Maria Gordina and Tai Melcher, A subelliptic Taylor isomorphism on infinite-dimensional Heisenberg groups, to appear in Probab. Theory Related Fields, (2011).

20. Mikhael Gromov, Carnot-Carathéodory spaces seen from within, Sub-Riemannian geometry, Progr. Math., vol. 144, Birkhäuser, Basel, 1996, pp. 79-323. MR1421823 (2000f:53034)

21. Leonard Gross, Potential theory on Hilbert space, J. Funct. Anal. 1 (1967), 123-181. MR0227747 (37:3331)

22. Martin Hairer and Jonathan C. Mattingly, Ergodicity of the 2D Navier-Stokes equations with degenerate stochastic forcing, Ann. of Math. (2) 164 (2006), no. 3, 993-1032. MR.2259251 (2008a:37095)

23. Lars Hörmander, Hypoelliptic second order differential equations, Acta Math. 119 (1967), 147-171. MR0222474(36:5526)

24. Svante Janson, Gaussian Hilbert spaces, Cambridge Tracts in Mathematics, vol. 129, Cambridge University Press, Cambridge, 1997. MR 1474726 (99f:60082)

25. Hui Hsiung Kuo, Gaussian measures in Banach spaces, Springer-Verlag, Berlin, 1975, Lecture Notes in Mathematics, Vol. 463. MR0461643 (57:1628)

26. Michel Ledoux, The geometry of Markov diffusion generators. Probability theory. Ann. Fac. Sci. Toulouse Math., (6) 9 (2000), no. 2, 305-366. MR1813804 (2002a:58045)

27. Peter $\mathrm{Li}$ and Shing-Tung Yau, On the parabolic kernel of the Schrödinger operator, Acta Math. 156 (1986), no. 3-4, 153-201. MR834612 (87f:58156)

28. Paul Malliavin, Hypoellipticity in infinite dimensions, Diffusion processes and related problems in analysis, Vol. I (Evanston, IL, 1989), Progr. Probab., vol. 22, Birkhäuser Boston, Boston, MA, 1990, pp. 17-31. MR.1110154(93b:60132)

29. Jonathan C. Mattingly and Étienne Pardoux, Malliavin calculus for the stochastic 2D NavierStokes equation, Comm. Pure Appl. Math. 59 (2006), no. 12, 1742-1790. MR2257860 (2007j:60082)

30. Tai Melcher, Heat kernel analysis on semi-infinite Lie groups, J. Funct. Anal. 257 (2009), no. 11, 3552-3592. MR2572261 (2011b:58074)

31. Richard Montgomery, A tour of subriemannian geometries, their geodesics and applications, Mathematical Surveys and Monographs, vol. 91, American Mathematical Society, Providence, RI, 2002. MR:1867362 (2002m:53045)

32. Hiroshi Sugita, Properties of holomorphic Wiener functions-skeleton, contraction, and local Taylor expansion, Probab. Theory Related Fields 100 (1994), no. 1, 117-130. MR.1292193 (96h:60092)

33. _ Regular version of holomorphic Wiener function, J. Math. Kyoto Univ. 34 (1994), 849-857. MR:1311623 (96b:60143) 
34. Feng-Yu Wang, Logarithmic Sobolev inequalities on noncompact Riemannian manifolds, Probab. Theory Related Fields 109 (1997), no. 3, 417-424. MR.1481127 (98i:58253)

35. _ Harnack inequality and applications for stochastic generalized porous media equations, Ann. Probab. 35 (2007), no. 4, 1333-1350. MR2330974 (2008e:60192)

36. - Harnack inequalities on manifolds with boundary and applications, J. Math. Pures Appl. (9) 94 (2010), no. 3, 304-321. MR2679029(2011f:58040)

Department of Mathematics, Purdue University, West Lafayette, Indiana 47907

E-mail address: fbaudoin@math.purdue.edu

Department of Mathematics, University of Connecticut, Storrs, Connecticut 06269 E-mail address: maria.gordina@uconn.edu

Department of Mathematics, University of Virginia, Charlottesville, Virginia 22903 E-mail address: melcher@virginia.edu 